\title{
In situ/operando synchrotron-based X-ray techniques for lithium-ion battery research
}

\author{
Seong-Min Bak', Zulipiya Shadike', Ruoqian Lin', Xiqian Yu ${ }^{2}$ and Xiao-Qing Yang ${ }^{1}$
}

\begin{abstract}
Lithium-ion battery (LIB) technology is the most attractive technology for energy storage systems in today's market. However, further improvements and optimizations are still required to solve challenges such as energy density, cycle life, and safety. Addressing these challenges in LIBs requires a fundamental understanding of the reaction mechanisms in various physical/chemical processes during LIB operation. Advanced in situ/operando synchrotron-based X-ray characterization techniques are powerful tools for providing valuable information about the complicated reaction mechanisms in LIBs. In this review, several state-of-the-art in situ/operando synchrotron-based X-ray techniques and their combination with other characterization tools for battery research are introduced. Various in situ cell configurations and practical operating tips for cell design and experimental set-ups are also discussed.
\end{abstract}

\section{Introduction}

Lithium-ion batteries (LIBs) are the most widely used electrochemical storage devices for portable electronics, and are considered the best candidates for the power systems of electrical vehicles ${ }^{1}$. Although extensive efforts have been made to develop LIBs, further improvement and optimization are still required, especially for energy density, cycle life, and safety characteristics.

Therefore, an in-depth understanding of the scientific aspects relating to LIBs, such as charge storage mechanisms, performance degradation mechanisms, and reactions at the electrode/electrolyte interfaces, is critically important. The synchrotron-based X-ray techniques provide powerful tools for studying and monitoring the changes of crystal structure, electronic structure, chemical composition, and morphology of electrode materials in electrochemical cells during operation, and the results are very valuable to improve the performance of existing systems and to design new battery materials.

Correspondence: Seong-Min Bak (smbak@bnl.gov) Xiqian Yu (xyu@iphy.ac.cn) or Xiao-Qing Yang (xyang@bnl.gov)

'Chemistry Division, Brookhaven National Laboratory, Upton NY11973, United States

${ }^{2}$ Beijing National Laboratory for Condensed Matter Physics, Institute of Physics, Chinese Academic of Science, Beijing 100190, P.R. China

These authors contributed equally: Seong-Min Bak, Zulipiya Shadike
Over the past few decades, significant developments in synchrotron-based X-ray techniques have provided substantial opportunities to study the working mechanisms of battery materials. When used in combination with other scattering, spectroscopy, and imaging techniques, comprehensive studies have been carried out to understand the complicated issues that arise during LIB operation with different physical/chemical aspects and at multiple length scales (Fig. 1 and Table 1). More importantly, highbrilliance synchrotron X-ray sources offer opportunities to perform in situ/operando experiments with various techniques to study dynamic properties and timedependent reaction process.

The significant advantages of in situ/operando approaches over ex situ characterization are as follows: (1) In situ measurements instantly probe the reaction taking place at the specific interested location of a sample, providing better reliability and higher precision for the data analysis; (2) in operando measurements continuously monitor electrochemical, physical, or chemical processes on a single sample or a device under operating conditions, eliminating the preparation of multiple samples for ex situ measurements and providing closer to real-time operation information; (3) this method allows the investigation of the non-equilibrium or fast-transient process during 


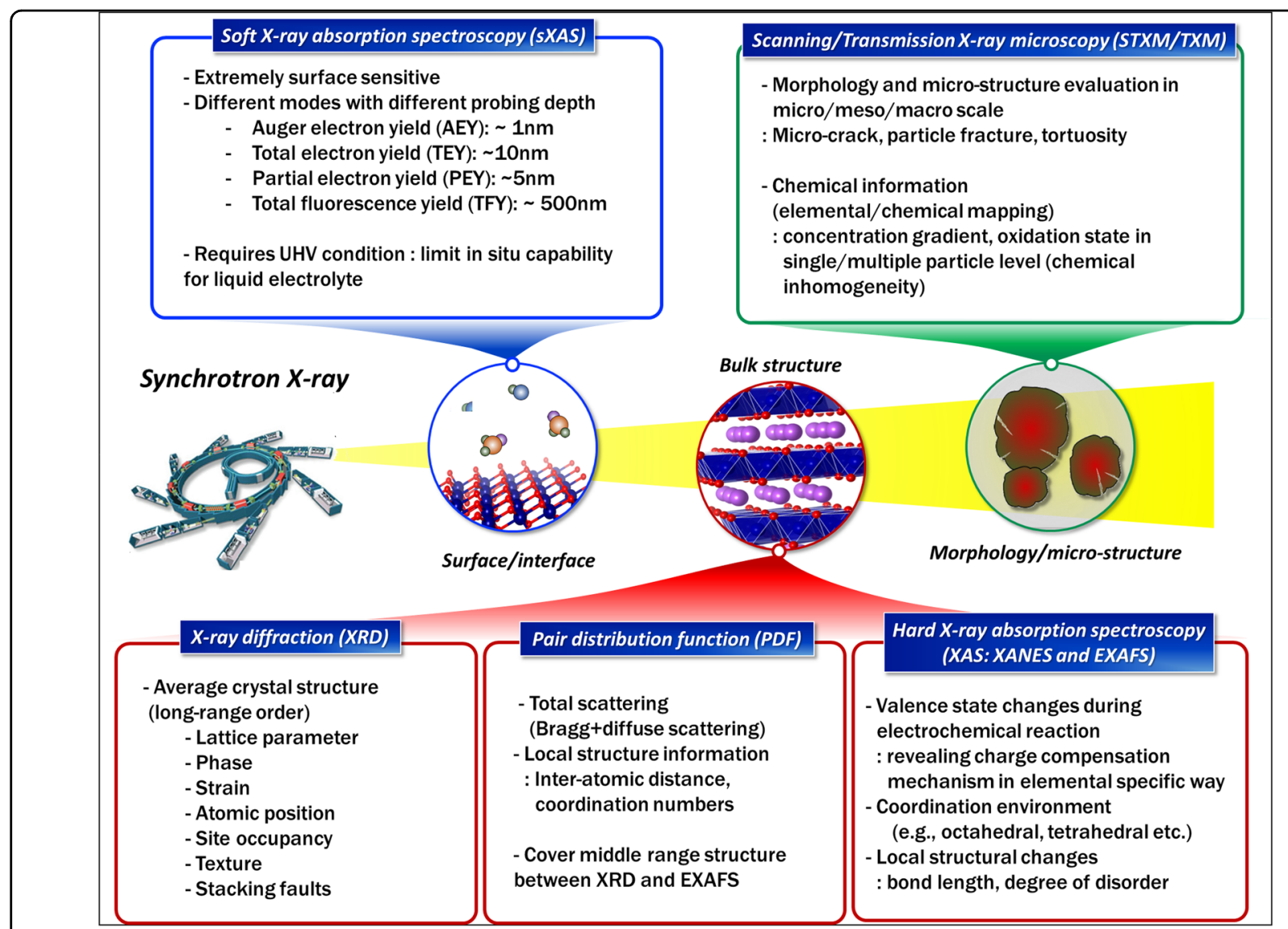

Fig. 1 Synchrotron based X-ray techniques. In situ synchrotron-based X-ray techniques used to study LIB materials and the information that obtained by each method

electrochemical or chemical reactions. The nonequilibrium or short-lived intermediate states or species, which cannot be captured by ex situ characterizations, can be detected ${ }^{2-5}$. (4) In situ approaches remove the possibility for contamination, relaxation, or irreversible changes of highly reactive charged/discharged samples when preparing, handling, and transferring them for ex situ measurements (e.g., the reaction with organic solvent during sample washing, the reaction with moisture and oxygen during sample transfer, etc. $)^{3}$, and the true reaction products can be more reliably identified. Indeed, the most recent results from advanced in situ/operando synchrotron-based experiments play a critical role in providing deeper insight into the complicated reaction mechanisms in LIBs ${ }^{6-10}$.

Considering the importance of advanced in situ/operando characterization techniques in battery studies, this review presents an overview of state-of-the-art in situ/ operando synchrotron-based X-ray techniques for LIB research, providing some basic guidelines for researchers in the battery field. In situ/operando techniques including $\mathrm{X}$-ray diffraction (XRD), X-ray pair distribution function
(PDF) analysis, X-ray absorption spectroscopy (XAS), and $\mathrm{X}$-ray imaging techniques will be discussed, and some practical tips for electrochemical cell design will be given.

\section{Designing in situ/operando X-ray characterization Combination of multiple in situ/operando X-ray techniques}

The electrochemical reactions of electrode materials usually involve complex, dynamic, and inhomogeneous processes, such as charge transfer, ion diffusion, formation and breakage of chemical bonding, phase transformation, and surface reconstruction. Due to the complex nature of the physical/chemical phenomena occurring during LIB operation, a combination of multiple complementary characterization techniques needs to be considered in order to provide more reliable information for understanding how these physical/chemical properties are related to the electrochemical performance of the batteries. Because each technique provides certain information about the different aspects of physical/chemical changes within the samples at different length scales, the choice and combination of appropriate techniques is quite important. 
Table 1 In situ synchrotron-based $X$-ray techniques and their capabilities/limitations

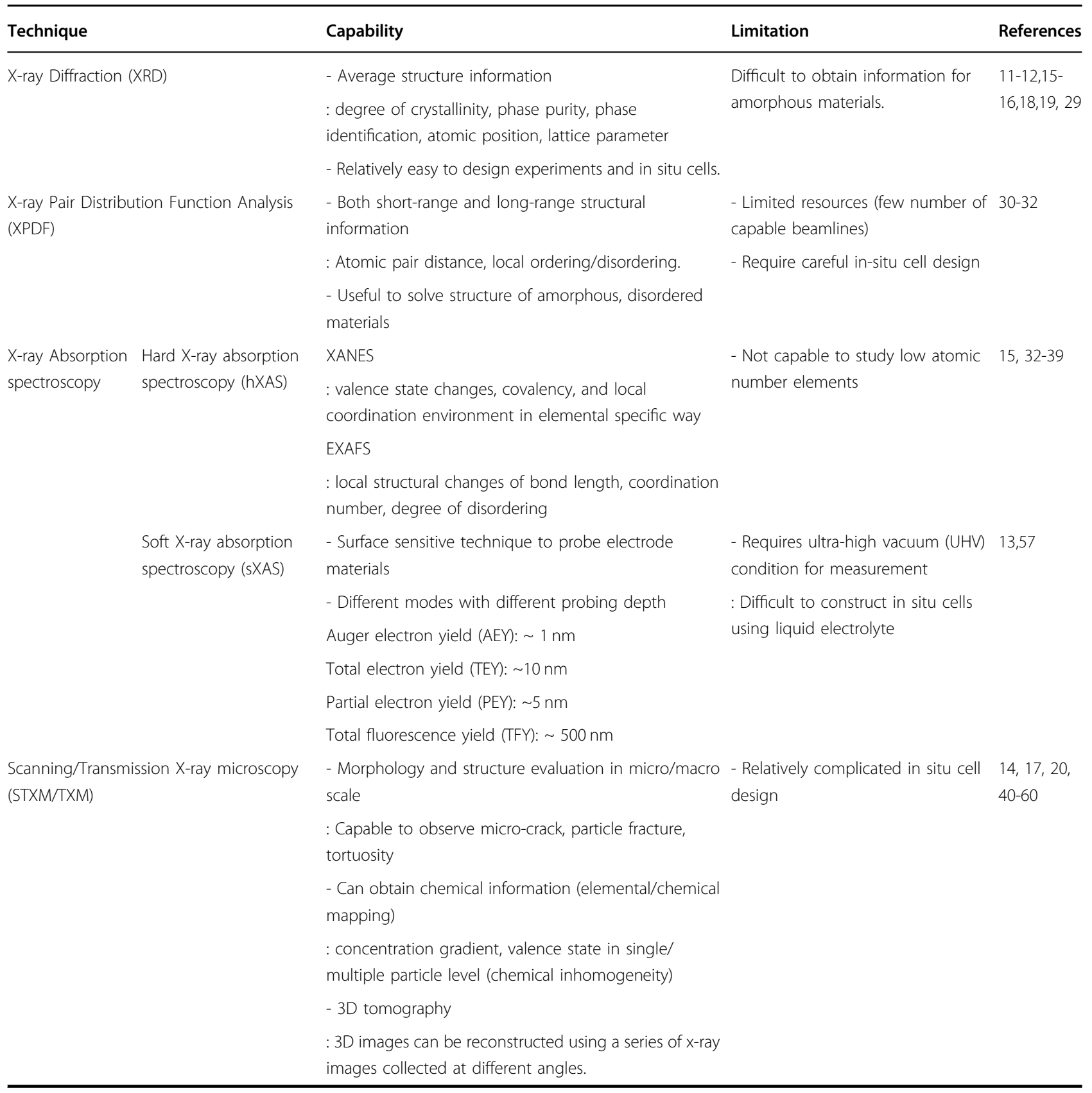

Since synchrotron beamtime is precious and difficult to get, in situ/operando experiments should be well planned and should focus only on dynamic processes for which ex situ measurements are inadequate. Therefore, ex situ measurements are still highly recommended prior to in situ/operando measurements. Ex situ results would provide some important information for designing more efficient in situ experiments. In particular, ex situ results can also be used as good references for in situ data analysis.

\section{In situ electrochemical cell design}

To conduct in situ experiments successfully, design of the in situ cell is the first important step. A poorly designed in situ cell could introduce artifacts into the data, and signals from inactive parts of the cell interfere with signals of interest. Therefore, the in situ cell needs to be designed carefully. In most cases, in situ cell should be designed to fit the specific experimental technique. Fortunately, many in situ cell designs for characterizing LIBs using various $\mathrm{X}$-ray techniques have recently been 


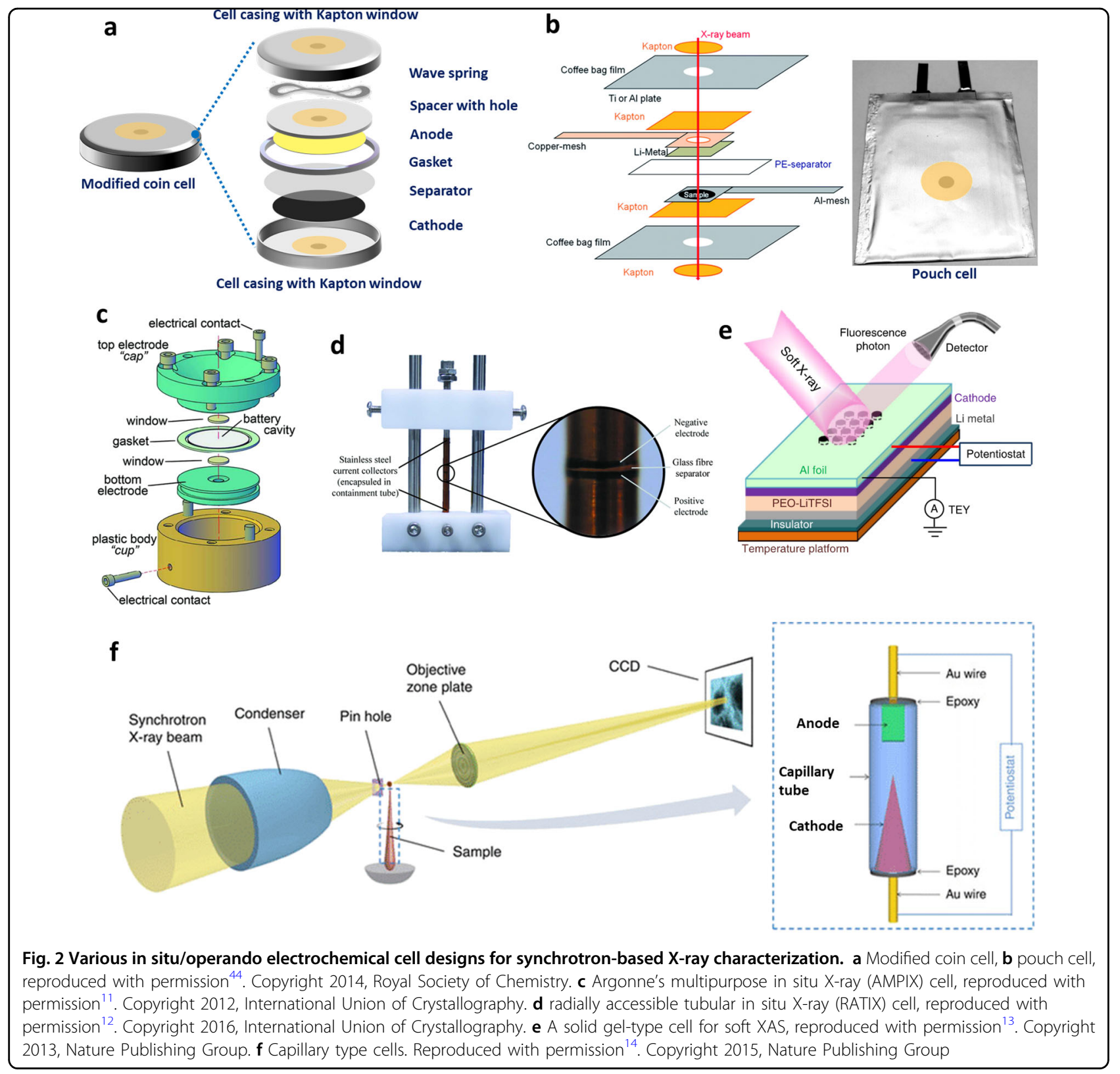

reported in the literature. As shown in Fig. 2, several different kinds of in situ/operando cells, such as modified coin cells, modified pouch cells, Argonne's multipurpose in situ X-ray (AMPIX) electrochemical cell ${ }^{11}$, radially accessible tubular in situ X-ray (RATIX) cell ${ }^{12}$, soft XAS cells $^{13}$, and capillary type cells ${ }^{14}$ have been reported for different types of in situ/operando experiments.

It is worthwhile to give some tips for designing in situ/ operando electrochemical cells as well as for the experimental design to make these cells function properly for both electrochemical processes and X-ray optics.

(1) The in situ/operando electrochemical cell should be designed to be easy to assemble/disassemble at synchrotron facilities. The cell should be highly reproducible for electrochemical testing. In addition, the cells should be able to be incorporated into the existing beamline setup and should fit properly in X-ray optics. For example, for in situ/ operando experiments with X-ray signal detection in reflection mode, an in situ/operando cell with a wide solid angle window for X-ray collection is often required. For in situ/operando X-ray tomography measurements, the sample needs to rotate in a wide range of angles to obtain a series of 2D images for the reconstruction of a 3D image. In this case, a cylindrical geometry and cell case made 
from low X-ray absorption materials are preferred to avoid blockage of outgoing X-rays ${ }^{11-13,15-19}$.

(2) To allow X-rays to reach the sample region and the detector, one hole for reflection mode and two holes for transmission mode are covered with X-ray transparent materials (e.g., Kapton film, beryllium, or aluminum foil, etc.) and are usually made on in situ/operando cells as windows, except for experiments using very high energy $\mathrm{X}$-rays, which can penetrate the entire cell body ${ }^{20}$. An ideal window material should be as follows: (a) chemically and electrochemically stable in the cell during operation. In other words, the window materials should not react with cell components, such as electrode and electrolyte, and should resist oxidation and reduction during charge and discharge; (b) impermeable to oxygen and moisture; (c) stiff to apply uniform pressure to ensure a uniform electrochemical reaction. Because of the penetration power of the beam is proportional to the X-ray energy, it is relatively easier to do in situ/operando experiments using hard X-rays due to more options for window materials. In general, polyimide (Kapton) film is a widely used window material for hard X-ray experiments not only because of its versatile performance, but also because it is very easy to handle. However, a thin Kapton film is too soft to apply constant pressure, causing the sample area underneath the window to react abnormally sometimes (e.g., no reaction, delay of the reaction, etc.). Beryllium should always be avoided due to safety hazards, and because it is easily oxidized during battery charging, though it is ideal for X-ray transmission. In the tender X-ray region, a very thin window with materials composed of light elements, such as thin polyester (Mylar) film, should be used ${ }^{21}$. Because ultra-high vacuum (UHV) environments are necessary for soft X-ray absorption spectroscopy (sXAS) measurements, an ultrathin silicon nitride window or solid-state electrolyte is frequently used for in situ sXAS experiments ${ }^{22}$.

(3) Interference from inactive cell components to outgoing X-ray signals should be avoided, especially for $X$-ray detection in transmission mode when the $\mathrm{X}$-ray beam has to penetrate the entire cell. Therefore, cell components or materials in the Xray beam path have to be carefully selected and constructed. For example, a Cu current collector, which is a conventionally used anode current collector, will cause attenuation of the X-rays and result in a poor signal-to-noise ratio $(\mathrm{S} / \mathrm{N})$ of the $\mathrm{X}$ ray signal detected when doing XAS experiments on low-Z elements (e.g., V, Cr, etc.). This could cause many problems when acquiring high-quality XAS data. Ti can be used to replace $\mathrm{Cu}$ as an anode current collector in such circumstances to improve the quantity of data. Interaction of the X-rays with cell components often generates background signals, which come together with X-ray signals from the real sample interested. For some measurements, such background signals need to be precisely removed afterwards from the total signals detected for quantitative data analysis. For example, data analysis of high-quality PDF requires both the Bragg scattering and diffuse scattering signals from only the sample. However, the background signals from other cell components, such as window materials, the separator, the current collector, the electrolyte, etc., collected and mixed together with the useful diffuse scattering signals from the sample need to be carefully removed. The background signals from an empty cell without the active sample need to be precisely measured and extracted during data collection and analysis. Therefore, the in situ/operando cells for PDF should be highly reproducible (e.g., exactly the same window materials, current collector, separator, same amount of electrolyte being used, etc.). An AMPIX cell (Fig. 2c) is capable of precisely measuring background signals and enabling reliable electrochemical performance ${ }^{11}$.

(4) Beam damage should also be considered. Unless continuous data collection is needed, intermittent sample probing (e.g., using beam shutter or moving the sample region off the beam, etc.) is recommended to reduce the total amount of X-ray radiation during the entire measurement.

More details on the data acquisition method, data processing program, and fitting method for each technique, can be found in the literature ${ }^{23-28}$.

\section{Synchrotron-based X-ray techniques for battery research} In situ X-ray diffraction (XRD)

The crystal structures of electrode materials are closely related to the properties of the battery cells including the power and energy density, cyclic life, and rate capability. In-depth understanding of these relationships is valuable to optimize the current electrode systems as well as develop new electrode materials with better electrochemical performance. Therefore, it is very important to study the structural changes of the electrode materials during the electrochemical process, including changes in the lattice parameters, phase transition, and atomic occupancy. In situ XRD has been proved as a very powerful technique for providing information about the 

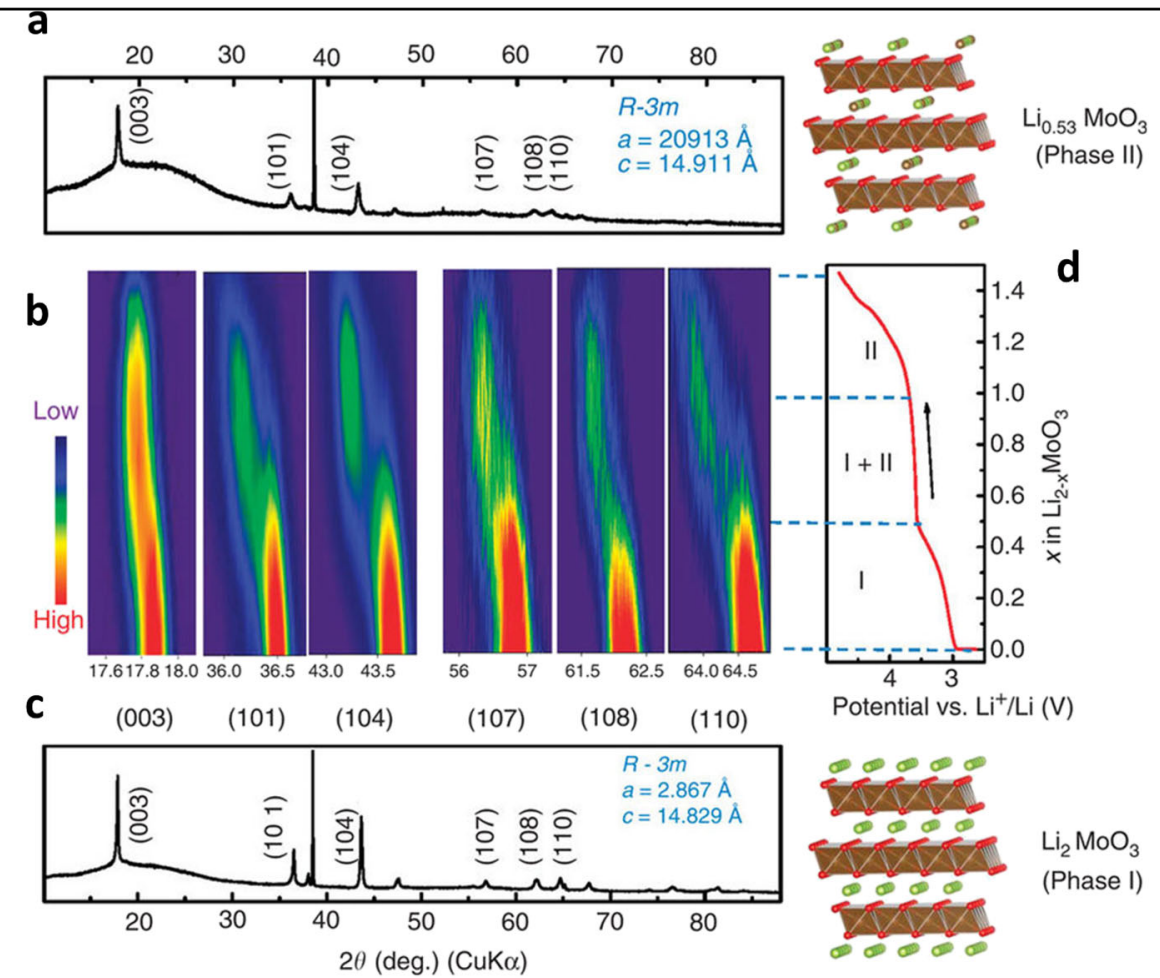

e

$0.1 \mathrm{C}$
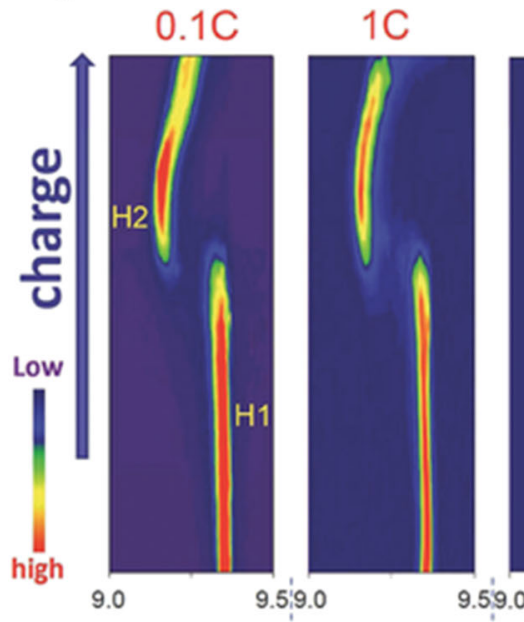

$10 \mathrm{C}$

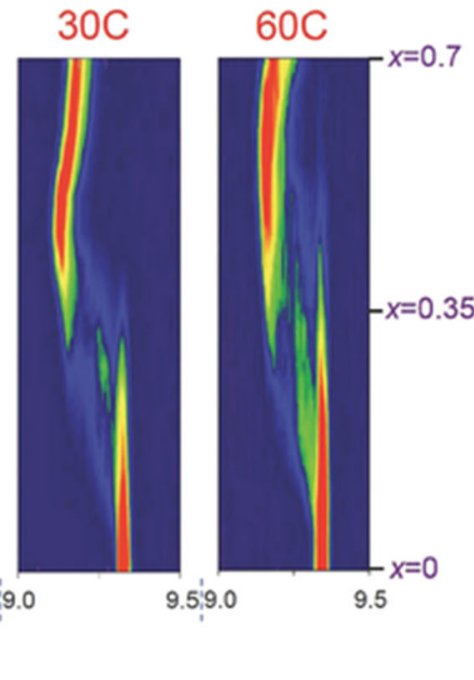

Fig. 3 In situ XRD of $\mathrm{Li}_{2} \mathrm{MoO}_{3}$ and TR-XRD of $\mathrm{LiNi}_{1 / 3} \mathrm{Co}_{1 / 3} \mathrm{Mn}_{1 / 3} \mathrm{O}_{2}$. a The XRD pattern of a $\mathrm{Li}_{2-x} \mathrm{MoO}_{3}$ electrode immediately after charging to $4.8 \mathrm{~V}$. b Contour plot of the diffraction peak evolution of the (003), (101), (104), (107), (108), and (110) planes during delithiation. c The XRD pattern of the $\mathrm{Li}_{2} \mathrm{MoO}_{3}$ electrode before charging. $\mathbf{d}$ Charge curve at a current density of $10 \mathrm{~mA} \mathrm{~g}^{-1}$ from the open-circuit voltage (OCV) to $4.8 \mathrm{~V}$ during XRD data collection; reproduced with permission ${ }^{29}$. Copyright 2014, Nature Publishing Group. e In situ XRD of $\mathrm{LiNi}_{1 / 3} \mathrm{Mn}_{1 / 3} \mathrm{CO}_{1 / 3} \mathrm{O}_{2}$ during the first charge. Contour plot of the (003) diffraction peak of $\mathrm{Li}_{1-x} \mathrm{Ni}_{1 / 3} \mathrm{CO}_{1 / 3} \mathrm{Mn}_{1 / 3} \mathrm{O}_{2}$ with as $x$ is increased between $x=0$ and 0.7 during the first charging process at different $C$ rates $\left(0.1,1,10,30\right.$, and 60 C). Data were collected at X14A at NSLS with a wavelength of $0.7747 \AA$. Reproduced with permission ${ }^{15}$. Copyright 2016, WILEY-VCH

crystal structural and phase transformation of crystalline electrode materials during cycling.

As a good example of in situ XRD studies, Zhou et al. ${ }^{29}$ investigated the structural evolution of a $\mathrm{Li}_{2} \mathrm{MoO}_{3}$ cathode material using in situ XRD, and proposed the "abnormal" unit cell breathing mechanism of $\mathrm{Li}_{2} \mathrm{MoO}_{3}$ during the charge-discharge process. As shown in Fig. 3a and $\mathrm{c}$, all the peaks in the XRD pattern of pristine and charged $\mathrm{Li}_{2} \mathrm{MoO}_{3}$ can be indexed based on a layered structure with the $R \overline{3} m$ space group. The in situ XRD 
patterns (Fig. 3b) of a $\mathrm{Li}_{2} \mathrm{MoO}_{3}$ electrode during the charge process demonstrated that the solid solution reaction occurs in the region of $0<x<0.5$ and all the main peaks shifted to lower angles, indicating an increase in $d$-spacing for phase I. Furthermore, a new phase (phase II), which has the same layered structure as phase I but larger lattice parameters formed during the charge process, suggests that a two-phase reaction occurred in region of $0.5<x<1$. Both lattice parameters $a(b)$ and $c$ of phase II were larger than those of phase I and increased continuously. In the $x>1$ region, phase I disappeared, and a solid solution reaction of phase II was observed. For widely used $\mathrm{LiMO}_{2}(\mathrm{M}=\mathrm{Co}, \mathrm{Ni}, \mathrm{Mn})$, lattice parameters, $a$ and $b$, contract during charging. However, for $\mathrm{Li}_{2} \mathrm{MoO}_{3}$, such changes are in opposite directions. Metal-metal bonding is used to explain such "abnormal" behavior and a generalized hypothesis is developed. The expansion of the $\mathrm{M}-\mathrm{M}$ bond becomes the controlling factor for $a(b)$ evolution during charge, which is in contrast to the shrinking $\mathrm{M}-\mathrm{O}$ bond as the controlling factor in "normal" materials.

Time-resolved X-ray diffraction (TR-XRD) techniques have also been developed to investigate the dynamic properties of electrodes during fast charge/discharge process. Taking advantages of the ultrafast data collection capability of TR-XRD using synchrotron X-ray beams. Zhou et al. ${ }^{15}$ also investigated the charge-rate dependent phase transition process of layered $\mathrm{LiNi}_{1 / 3} \mathrm{Mn}_{1 / 3} \mathrm{Co}_{1 / 3} \mathrm{O}_{2}$ by using fast in situ TR-XRD. As shown in Fig. 3e, the charging process at current rates of 0.1 and $1 \mathrm{C}$ show two regions of solid solution reactions with two hexagonal phases of $\mathrm{H} 1$ to $\mathrm{H} 2$ and the two-phase coexistence region of $\mathrm{H} 1+\mathrm{H} 2$ in the middle stage of the charging process. Interestingly, when the charging rate is increased to $10 \mathrm{C} \mathrm{a}$ new intermediate phase was observed in between the two solid solution reaction regions, and the peaks representing this new phase become more and more pronounced at higher rates of 30 and $60 \mathrm{C}$. Furthermore, an abnormal Lipoor region with tetrahedral $\mathrm{Li}$ occupation and a normal Li-rich region with octahedral Li occupation was observed in the half-charged sample using scanning transmission electron microscopy (STEM). It is suggested that the intermediate phase can act as a buffer between the Li-rich and Li-poor phase and can reduce the local stress and strain produced by inhomogeneity during charging.

Like in the case of the above examples, modified coin cells (Fig. 2a) are used for in situ XRD measurements. However, other cases, flexible Kapton tape windows cannot provide a sufficiently airtight seal and cannot keep out moisture for a long time. The potential for leakage of electrolyte from the cell is a large problem. In addition, the cell casing with holes (windows) can cause uneven pressure and windows using insulating materials can reduce the electrical connection with the electrode. To overcome these drawbacks of the coin cell, an AMPIX cell (Fig. 2c) with a conductive glassy carbon window was designed by Borkiewicz et al. ${ }^{11}$ This cell has been proved as compatible with various synchrotron-based X-ray scattering and spectroscopy measurements without the undesired delays in the electrochemical reactions near the window area and shows improved long-term cycle stability. Liu et al. ${ }^{16}$ investigated the dynamic process of the $\mathrm{LiFePO}_{4}$ electrode material by using TR-XRD with an AMPIX cell and detected the metastable intermediate phase. At lower current rates of 5 and $10 \mathrm{C}$, all diffraction peaks observed from the in situ XRD of the initial 5 cycles can be indexed to either the $\mathrm{Li}$-rich $\mathrm{Li}_{1-\alpha} \mathrm{FePO}_{4}$ or Li-poor $\mathrm{Li}_{\beta} \mathrm{FePO}_{4}$ phase. Surprisingly, a new phase with lattice parameters smaller than those of $\mathrm{LiFePO}_{4}$ but larger than those of $\mathrm{FePO}_{4}$ under equilibrium was observed at a fast rate of $20 \mathrm{C}$. The individual diffraction patterns during the first cycle at various rates of 5,10 , and $20 \mathrm{C}$ were also compared. The results, as shown in Fig. $4 \mathrm{a}-\mathrm{c}$, demonstrated that the phase transformation in $\mathrm{LiFePO}_{4}$ at a high rate continues by changing the structure rather than through distinct relocation of the phase boundary between $\mathrm{LiFePO}_{4}$ and $\mathrm{FePO}_{4}$. This kind of structural change avoids a major structural rearrangement across a moving interface, which helps to explain the good highrate performance of $\mathrm{LiFePO}_{4}$ despite a large Li miscibility gap between $\mathrm{LiFePO}_{4}$ and $\mathrm{FePO}_{4}$ at room temperature. This work also reconfirmed that investigation of the equilibrium system (e.g., ex situ measurement) is insufficient to elucidate the operation mechanism of electrode materials operated at high rates.

Most in situ cells including coin cells, pouch cells, and AMPIX cells are designed for an X-ray geometry, so the incident beam is along the electrode stacking direction. In this measurement geometry, the incident beam penetrates all the cell components such as the cathode, anode, separator, and electrolytes, and the diffracted signals from these components are collected by the detector. The advantages of this geometry is the easy construction and alignment of the in situ cell, but a disadvantage is the inability to distinguish the signals coming from different parts of the electrode sample along the beam path. In other words, if structural changes are inhomogeneous among the parts near the current collector and closer to the separator, they will not be individually detected. As we know, inhomogeneous electrochemical reactions should be considered for cathode materials, especially when thick electrodes are used. Thicker electrodes can increase the energy density of the cell, but may cause more serious inhomogeneous reactions, especially at high cycling rates. To investigate the rate dependent inhomogeneous reactions in parts near current collector to those close to the separator, a RATIX cell (Fig. 2d) for spatially resolved in operando X-ray scattering and spectroscopic studies was 


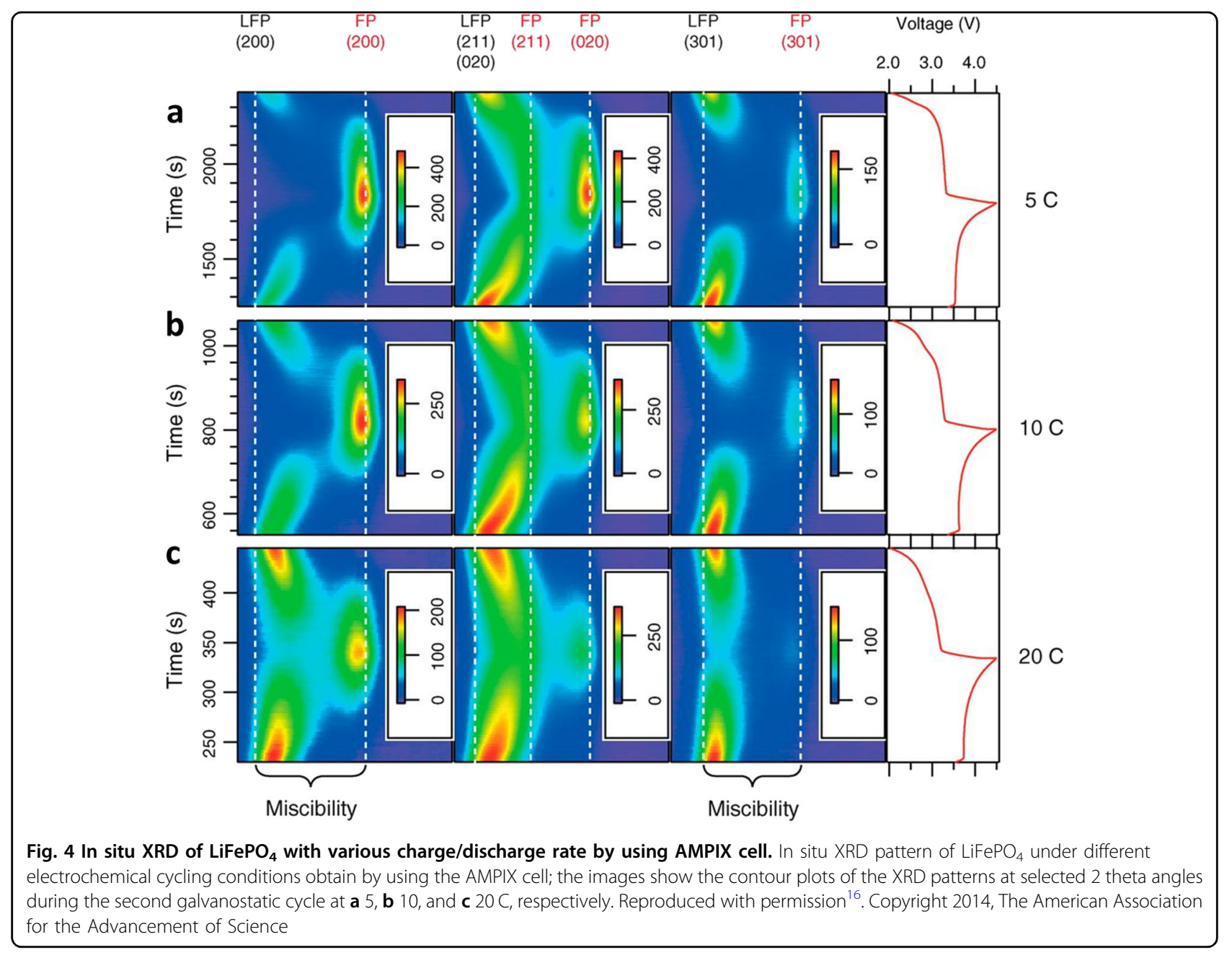

developed with advantages of the high intensity and micron focused X-ray source from the Advanced Photon Source (APS) beamline. Liu et al. ${ }^{12}$ conducted a "depth profile" measurement of the $\mathrm{LiFePO}_{4}$ electrode and provided insight into the relationship between the inhomogeneity of the structural changes and the electrochemical performance of the cell by using the RATIX cell. The important conclusion is that the electrode parts near the separator showed a more advanced reaction than those near the current collector under the same charge (discharge) state.

\section{In situ pair distribution function (PDF)}

Although XRD techniques can provide important structural information about crystalline materials, it is not easy to perform characterization studies using conventional powder diffraction on materials without long-range order, such as amorphous materials or materials with nano-sized crystals. For instance, it is hard to investigate the reaction mechanism of conversion-type electrode materials using XRD due to nano-sized and highly disordered particles that form during the conversion-type electrochemical process. For this kind of study, the pair distribution function (PDF) is a good choice. While XRD covers only Bragg scattering and provides long-range average structural information, the PDF techniques utilize the total scattering (Bragg and diffuse scattering), which can be used to investigate the materials with short-range ordering, and provides local information especially the atomic pair distribution relating to chemical, structural, and morphological transformations that occur during electrochemical reactions.

Mixed-anion iron oxyfluoride (i.e., FeOF) material is a typical conversion-type electrode with a good rate capability and relatively good reversibility. Wiaderek et al. ${ }^{30}$ investigated the electrochemical reaction mechanism of an FeOF electrode using in operando PDF combined with Li nuclear magnetic resonance (NMR) NMR spectroscopy. For better background subtraction, an AMPIX cell was used in this in situ PDF experiment, 

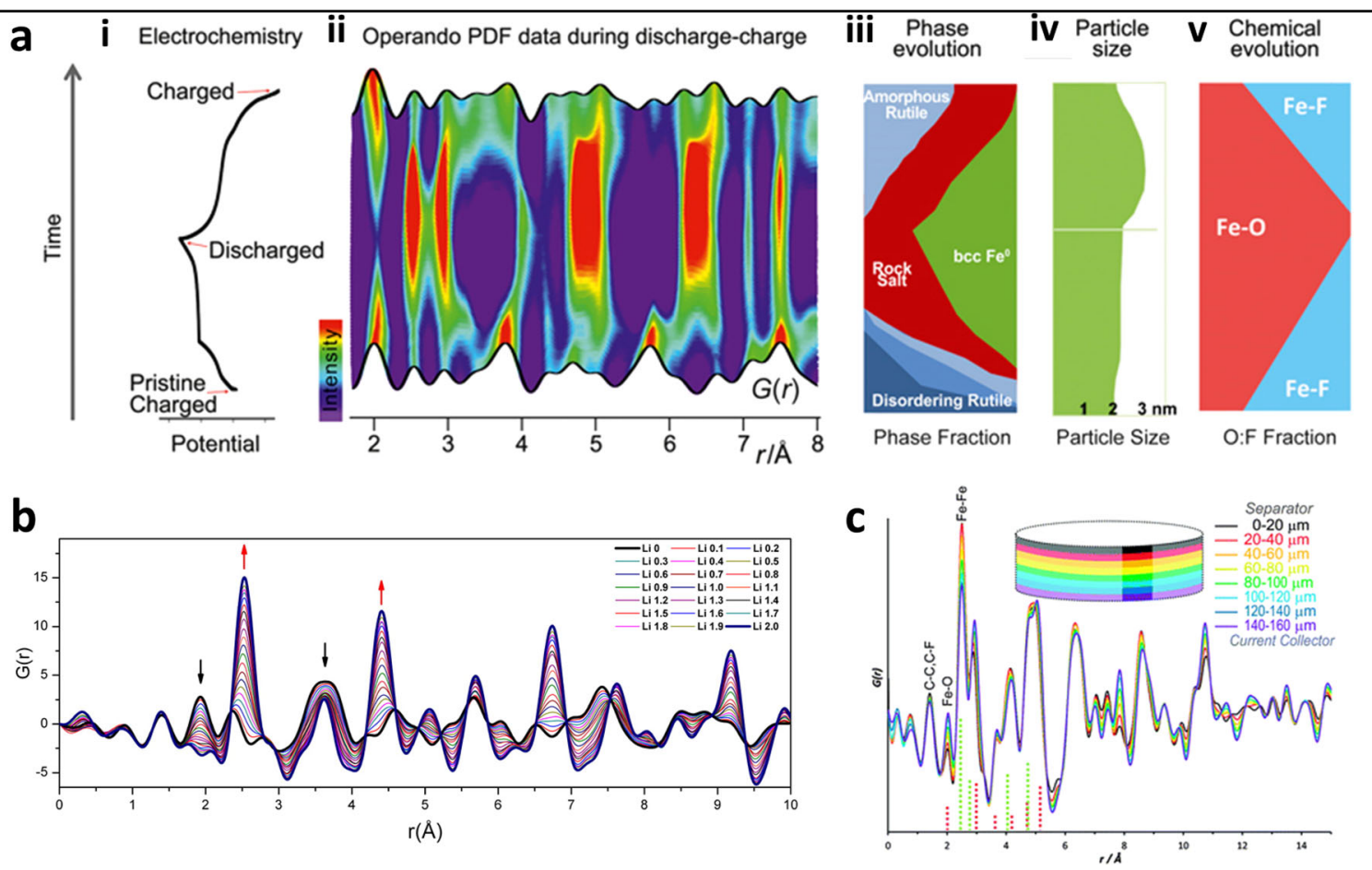

Fig. 5 In situ PDF and depth profile study of conversion type electrode materials. a (i) The electrochemical lithiation (discharge) and delithiation (charge) curve of an FeOF electrode. (ii) A series of PDF data can be obtained at fine reaction intervals (0.1 Li steps here). Structural models can be fitted to the data to show how (iii) the phases and (iv) particle size evolve during the reaction, or (v) the changes in the Fe-O and Fe-F features can be evaluated to show how the chemistry changes. Reproduced with permission ${ }^{30}$. Copyright 2016, Cambridge University Press. b In situ PDFs for $\mathrm{CCN}_{-} \mathrm{CuF}_{2}$ on the first discharge with the initial and end states highlighted in bold. Black and red arrows indicate a decrease and increase in intensity, respectively. Reproduced with permission ${ }^{31}$. Copyright 2014, American Chemical Society. c A schematic of the layered architecture typical of a LIB and the PDF data obtained for various depths within the electrode. Reproduced with permission ${ }^{32}$. Copyright 2013, Royal Society of Chemistry

and the results are shown in Fig. 5a. The PDF data collected during the initial discharge process show smooth changes in local structural features and bond lengths, indicating the conversion of FeOF to metallic Fe. Rietveld refinement results show the transformation from $\mathrm{FeOF}$ to Fe via a rock-salt-type intermediate phase. More interestingly, the structure of the re-charged electrode is completely different from the initial single-phase electrode, although the recharge capacity is almost fully reversible for the discharge capacity. By analyzing the bond length, oxidation state, and coordination numbers of the positions and the intensities of the peaks representing the $\mathrm{Fe}-\mathrm{O} / \mathrm{F}$ and $\mathrm{Fe}-\mathrm{Fe}$ pairs, the charge capacity should be attributed to the formation of a nanoscale composite of the rock-salt phase, which is oxide-rich, and the rutile phase, which is fluoride-rich. Similarly, Hua et al. $^{31}$ investigated the reaction mechanism of a conversion-type $\mathrm{CuF}_{2}$ electrode using PDF and NMR measurements, and provided the results of the local structure information during the first discharge process. As shown in Fig. 5b, the PDF peaks of equatorial and axial $\mathrm{Cu}-\mathrm{F}$ bonds in the pristine electrode indicate Jahn-Teller distortion of $\mathrm{Cu}^{2+}$. Variations in the peak position and intensity during the discharge process suggest the transformation from $\mathrm{CuF}_{2}$ to metallic $\mathrm{Cu}$.

PDF is also utilized for the depth profile measurement to investigate inhomogeneous structural changes at the electrode level. Unfortunately, few reports using in situ/ operando PDF to do depth profile studies have been conducted, and we believe such studies will provide more in-depth understanding of the inhomogeneity of electrochemical reactions in the near future. Borkiewicz et al. ${ }^{32}$ designed an ex situ experiment to investigate the distribution of the charge/discharge states perpendicular to the electrode surface by using spatially resolved hard Xray PDF. A free-standing pellet of nanoscale $\alpha-\mathrm{Fe}_{2} \mathrm{O}_{3}$ was used as an electrode, and was discharged to the midpoint of the fully discharged state during conversion of the rock-salt phase to the Fe nanoparticle phase. PDF data collected as a function of distance from the surface of the partially discharged electrode are shown in Fig. 5c. The features associated with $\mathrm{Fe}(\mathrm{Fe}-\mathrm{Fe} \sim 2.5 \AA)$ are most pronounced near the surface, while the features associated with the rock-salt phase $\left(\mathrm{Fe}_{1-x} \mathrm{O}, \mathrm{F} m 3 m, a=4.2 \AA\right)$ are most pronounced near the current collector. Phase 


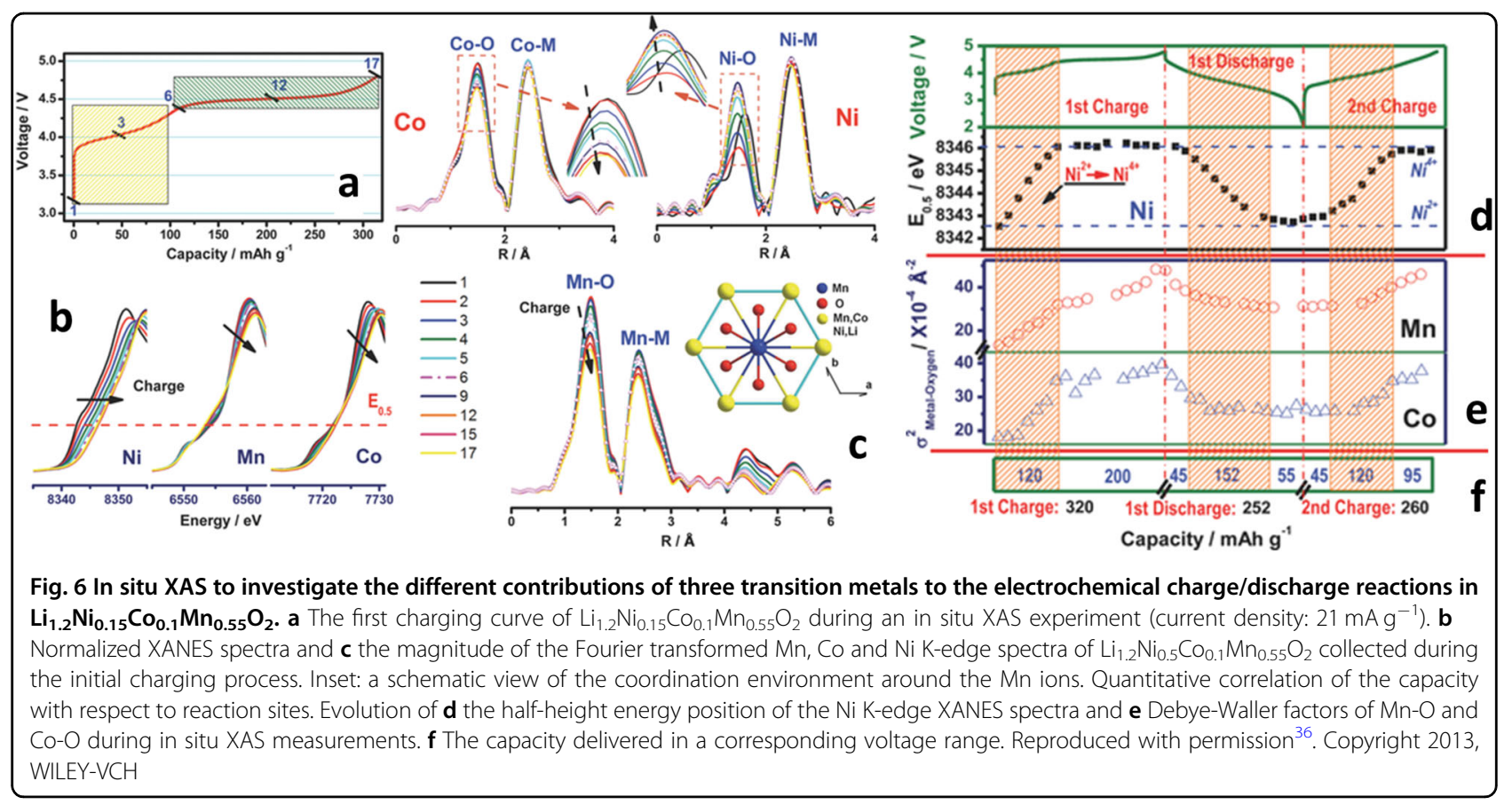

fraction analysis (Fig. 5d) suggested that the reaction is most advanced close to the separator, and the rate is limited by Li diffusion rather than electronic conductivity. In this case, a higher current rate may exacerbate the formation of a phase fraction gradient.

\section{In situ X-ray absorption spectroscopy (XAS)}

XAS is a technique that measures the X-ray absorption coefficient of a sample as a function of the X-ray photon energy below and above the absorption edge of a certain element. According to the energy range (wavelength) of the probing X-ray source, XAS can be divided into soft $(<1 \mathrm{keV})$, tender $(1-5 \mathrm{keV})$, and hard XAS $(>5 \mathrm{keV})^{33}$.

An XAS spectrum is typically divided into two regions by the relative energy range to the absorption edge: (1) the X-ray absorption near-edge structure (XANES), which covers from a few $\mathrm{eV}$ below the absorption edge to approximately $50 \mathrm{eV}$ above the edge, and (2) the extended $\mathrm{X}$-ray absorption fine-structure (EXAFS), which extends from approximately $50 \mathrm{eV}$ above the edge to as much as $1000 \mathrm{eV}^{34}$. The XANES data collected on the cathode samples cycled to different states of charge provide useful quantitative or qualitative information about the changes in the oxidation states of the elements being probed, their site symmetries, and the covalent bond strengths. EXAFS can be used to study short-range local structures and obtain coordination numbers, bond distances, and the chemical identity of the nearest neighbors through data fitting. In general, for soft XAS, the EXAFS part is not considered, and the typical data are collected on the $\mathrm{K}$ edges of low-Z elements (e.g., $\mathrm{C}, \mathrm{N}, \mathrm{O}$ ) and L-edges of $3 d$ transition metals ( $3 d-\mathrm{TM})$. XANES in soft XAS is also called near-edge X-ray absorption fine-structure (NEXAFS) spectroscopy ${ }^{35}$.

A great advantage of XAS over other techniques is the elemental selectivity, which enables us to study the local chemical environment surrounding certain elemental atoms. Due to its elemental selectivity and high chemical sensitivity, in situ XAS has been used as a key characterization tool to study the charge compensation mechanisms of LIB electrode materials. In situ XAS can provide information about the chemical environment and oxidation state of each individual element in a material containing multiple elements, helping us to determine the main contributing element of a redox processes at a certain charge/discharge state.

A good example of this is the work of Yu et al..$^{36}$, which studied different contributions of three transition metals to the electrochemical charge/discharge reactions in $\mathrm{Li}_{1}$. ${ }_{2} \mathrm{Ni}_{0.15} \mathrm{Co}_{0.1} \mathrm{Mn}_{0.55} \mathrm{O}_{2}$. As shown in Fig. 6a, $\mathrm{Li}_{1.2} \mathrm{Ni}_{0.15^{-}}$ $\mathrm{Co}_{0.1} \mathrm{Mn}_{0.55} \mathrm{O}_{2}$ exhibits a high-charge capacity of $300 \mathrm{mAhg}^{-1}$, with a high average operating voltage $\left(>3.5 \mathrm{~V}\right.$ vs. $\left.\mathrm{Li} / \mathrm{Li}^{+}\right) . \mathrm{Ni}, \mathrm{Co}$, and $\mathrm{Mn}$ K-edge XANES (Fig. 6b) shows that major charge compensation at the metal site during charging is achieved by the oxidation of $\mathrm{Ni}^{2+}$ ions, as reflected by the rigid edge shift of $\mathrm{Ni}$, while the K-edge positions of $\mathrm{Mn}$ and Co ions remain mostly unchanged near the $\mathrm{Mn}^{4+}$ and $\mathrm{Co}^{3+}$ edge positions. Since the K-edge XANES energy position changes can only be used to track the contribution from $\mathrm{Ni}$ but not from $\mathrm{Mn}$ and $\mathrm{Co}$, other spectral parameters are needed to correlate the structural changes with the charge capacity 

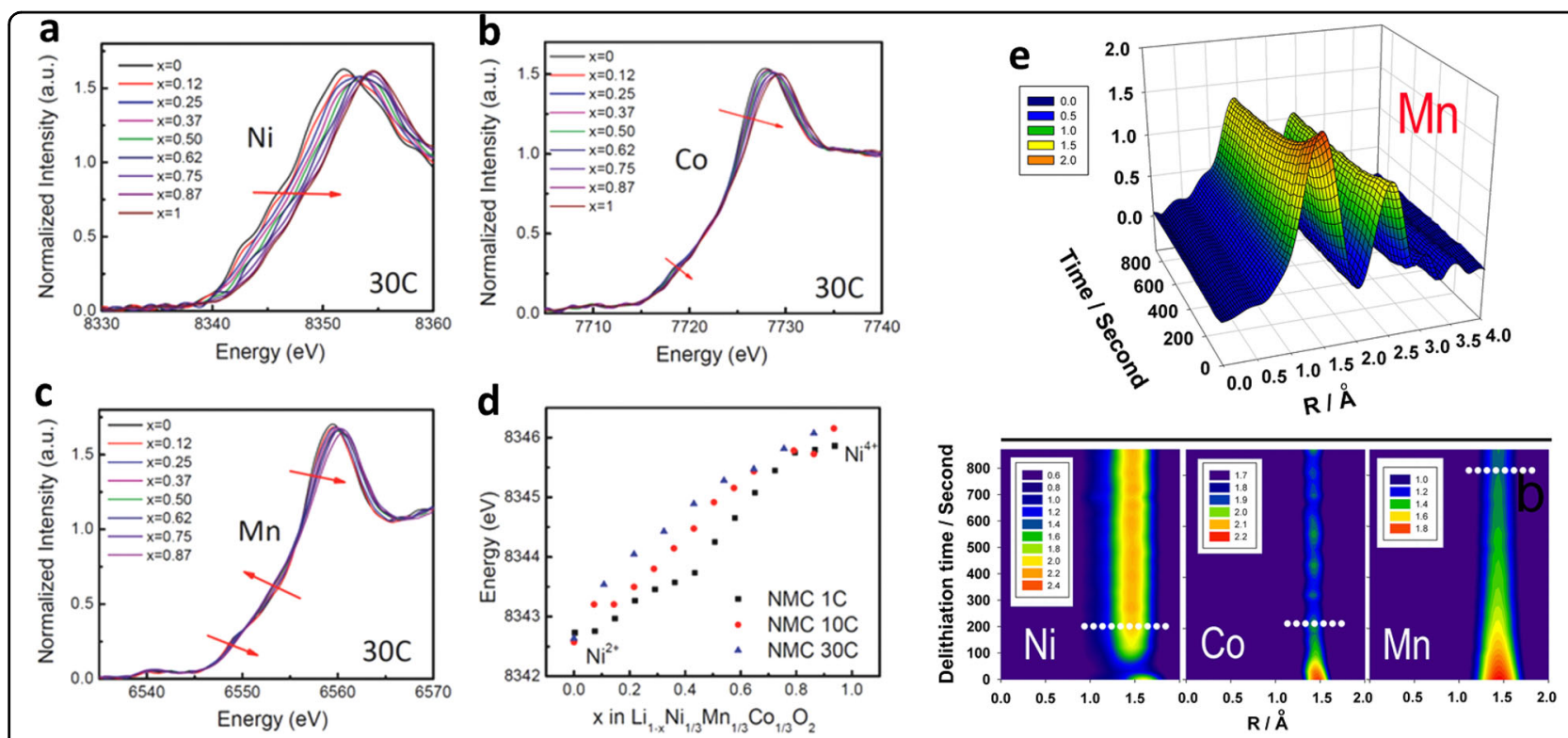

Fig. 7 In situ TR-XAS of $\mathrm{LiNi}_{1 / 3} \mathrm{Mn}_{1 / 3} \mathrm{Co}_{1 / 3} \mathrm{O}_{2}$ and $\mathrm{Li}_{1.2} \mathrm{Ni}_{0.15} \mathrm{Co}_{0.1} \mathrm{Mn}_{0.55} \mathrm{O}_{2}$ during constant current charging and constant voltage charging. Normalized XANES spectra of NMC at the $\mathbf{a} \mathrm{Ni}, \mathbf{b} \mathrm{Co}$, and $\mathbf{c} \mathrm{Mn}$ K-edges during $30 \mathrm{C}$ charging. $\mathbf{d}$ The Ni K-edge energy shift as a function of nominal lithium content $x$ in $\mathrm{Li}_{1-x} \mathrm{Ni}_{1 / 3} \mathrm{Mn}_{1 / 3} \mathrm{CO}_{1 / 3} \mathrm{O}_{2}$ during the initial charge process at the current rates of 1, 10, and $30 \mathrm{C}$. Reproduced with permission ${ }^{15}$. Copyright 2016, WILEY-VCH. e Magnitude of the Fourier transformed Mn K-edge spectra of $\mathrm{Li}_{1.2} \mathrm{Ni}_{0.15} \mathrm{Co}_{0.1} \mathrm{Mn}_{0.55} \mathrm{O}_{2}$ collected during charging with a $5 \mathrm{~V}$ constant voltage. The projection view of the corresponding $\mathrm{Ni}-\mathrm{O}, \mathrm{Co}-\mathrm{O}$, and $\mathrm{Mn}-\mathrm{O}$ peak magnitudes of the Fourier transformed $\mathrm{K}$-edge spectra as a function of the charging time. Reproduced with permission ${ }^{36}$. Copyright 2013, WILEY-VCH

contribution of $\mathrm{Mn}$ and Co during the charging process. EXAFS analysis was performed to correlate the charge capacity with changes in the intensity of the $\mathrm{Mn}-\mathrm{O}$ and $\mathrm{Co}-\mathrm{O}$ first shell peaks obtained by Fourier transformed EXAFS spectra (Fig. 6c). Semi-quantitative analysis of Ni K-edge XANES with energy position changes and the Debye-Waller factors of $\mathrm{Mn}-\mathrm{O}$ and $\mathrm{Co}-\mathrm{O}$ derived from EXAFS fitting during cycling were also performed, as presented in Fig. $6 \mathrm{~d}$ and e. The $\sigma_{\mathrm{Mn}-\mathrm{O}}$ values show a continuous increase in the whole voltage range up to $4.8 \mathrm{~V}$ during the initial charge process. Changes in these values before the voltage plateau (approximately $4.5 \mathrm{~V}$ ) could be caused by local structural changes induced by lithium extraction from the $\mathrm{LiMO}_{2}$ structure. The $\sigma_{\mathrm{Co}-\mathrm{O}}$ values show a significant increase in the first voltage slope region $(3-4.5 \mathrm{~V})$. Upon further charging in the voltage plateau region, the $\sigma_{\mathrm{Mn}-\mathrm{O}}$ values continue to increase, reaching the maximum value at the end of the first charge, while the $\sigma_{\mathrm{Co}-\mathrm{O}}$ and $\sigma_{\mathrm{Ni}-\mathrm{O}}$ values reveal only a slight change, indicating that the lithium extraction-induced local structural changes mainly occur in the area surrounding the $\mathrm{Mn}$ sites, thus providing more information about the contribution of $\mathrm{Mn}$.

The dispersive XAS instrumentation enables timeresolved XAS (TR-XAS) experiments on a time scale as short as milliseconds ${ }^{37,38}$, making it possible to investigate the dynamic process of battery materials under high-rate operating conditions. Unlike conventional in situ XAS experiments performed at slow charge/discharge rates (e.g., $\mathrm{C} / 20$ and $\mathrm{C} / 5$ ) for several hours, Zhou et al. ${ }^{15}$ reported results collected in a time frame as short as several minutes at very high charge-discharge rates utilizing the TR-XAS technique. Figure $7 \mathrm{a}-\mathrm{c}$ shows the $\mathrm{Ni}, \mathrm{Co}$, and $\mathrm{Mn} \mathrm{K}$-edge XANES spectra of $\mathrm{LiNi}_{1 / 3} \mathrm{Mn}_{1 / 3} \mathrm{Co}_{1 / 3} \mathrm{O}_{2}$ during $30 \mathrm{C}$ charging (charged in $2 \mathrm{~min}$ ). To avoid potential problems that could be caused by soft Kapton windows, the modified coin cell using glassy carbon windows was used to enhance the pressure of the window region. The K-edge of Ni shifts continuously to higher energy, indicating oxidation of $\mathrm{Ni}$ ions from $\mathrm{Ni}^{2+}$ to $\mathrm{Ni}^{4+}$ during the charging process. In contrast, the K-edges of Co and Mn show almost no energy shift. Only changes to the shape of the Co and Mn K-edge features occur, indicating that the local environment changes around the Co and $\mathrm{Mn}$ ions. However, Co and $\mathrm{Mn}$ contribute very little to the capacity. The edge shift of Ni Kedge XANES as a function of the state-of-charge (amount of delithiated $\mathrm{Li}^{+}$) at different $\mathrm{C}$ rates (Fig. 7d) demonstrates that oxidation of $\mathrm{Ni}$ accelerates with increasing charging rate, and the $\mathrm{Ni}^{4+}$ state can be reached at the end of charging even at a high $30 \mathrm{C}$ rate. These results show the good kinetic properties of $\mathrm{Ni}$, indicating that the high $\mathrm{Ni}$ content in NMC aids in achieving a high rate capability. Another good example of TR-XAS studies is the work of $\mathrm{Yu}$ et $\mathrm{al}^{36}$, which aimed to better understand the rate-limiting element in $\mathrm{Li}_{1.2} \mathrm{Ni}_{0.15} \mathrm{Co}_{0.1} \mathrm{Mn}_{0.55} \mathrm{O}_{2}$. Yu et al. applied a constant voltage of $5 \mathrm{~V}$ on a $\mathrm{Li}_{1.2} \mathrm{Ni}_{0.15} \mathrm{Co}_{0.1} \mathrm{Mn}_{0.55} \mathrm{O}_{2}$ 

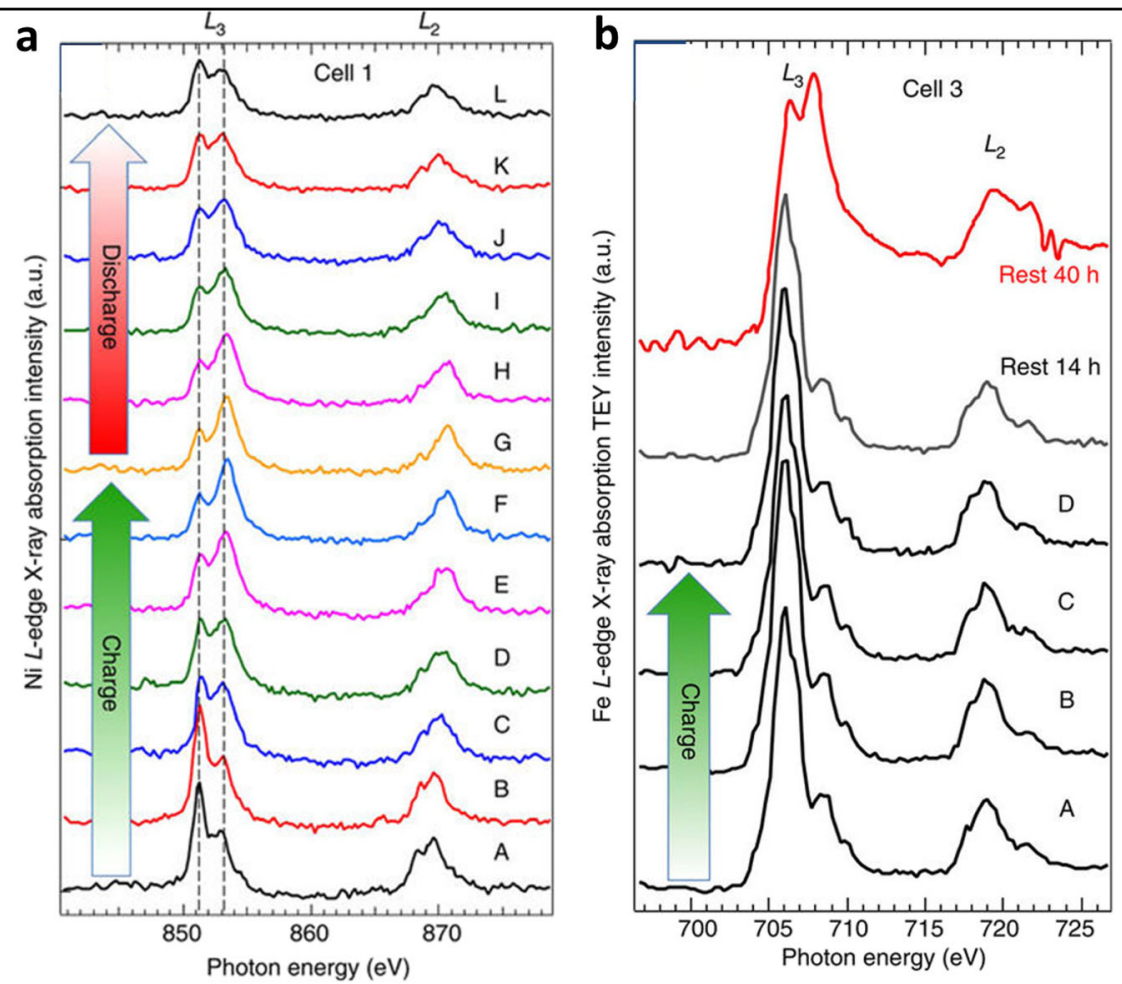

Fig. 8 Operando sXAS results collected by using a LiNi ${ }_{1 / 3} \mathrm{Mn}_{1 / 3} \mathrm{Co}_{1 / 3} \mathrm{O}_{2} /$ PEO-LiTFSI/Li battery cell. a Ni L-edge sXAS TFY spectra of an NMC cathode. Data collected simultaneously with electrochemical cycling at a C/5 rate. b Fe L-edge SXAS TEY spectra of the LiFePO $\mathrm{C}_{4}$ cathode in Cell 3 at different electrochemical cycling stages labeled $A-D$ in the initial charge cycle and after 14 and $40 \mathrm{~h}$ of relaxation. The line shape at $\mathrm{L}_{3}$ at approximately $706 \mathrm{eV}$ and $\mathrm{L}_{2}$ at approximately $719 \mathrm{eV}$ fingerprints the oxidation state of Fe in $\mathrm{LiFePO}$, which does not show any change without long relaxation. Reproduced with permission ${ }^{13}$. Copyright 2013, Nature Publishing Group

cathode to oxidize $\mathrm{Ni}, \mathrm{Co}$, and $\mathrm{Mn}$ ions simultaneously. The comparative Fourier transformed magnitude of the corresponding $\mathrm{Ni}$, Co, Mn K-edge EXAFS spectra shown in Fig. 7e demonstrated the different kinetic behaviors of the three metal elements. The results clearly showed that oxidation of $\mathrm{Mn}$ is much slower than oxidation of $\mathrm{Ni}$ and Co in $\mathrm{Li}_{1.2} \mathrm{Ni}_{0.15} \mathrm{Co}_{0.1} \mathrm{Mn}_{0.05} \mathrm{O}_{2}$ cathode materials, and nano-domains of $\mathrm{Li}_{2} \mathrm{MnO}_{3}$ are the major rate-limiting factors.

Compared with hard XAS, sXAS is a more direct and efficient tool to probe the electronic states through excitation of core-level electrons to unoccupied states above the Fermi level. In particular, for LIB research, $3 d$ electron states of $3 d-\mathrm{TM}$ and the $p$ electron states of anions can be directly studied using sXAS. In addition, sXAS can be used to study samples with various probing depths (from surface to bulk), by using different signal detection modes (as shown in Fig. 1). Due to the different penetration lengths of the photo electrons and fluorescence photons, the electron yield (EY) mode signal mainly provides information about the surface $(1-10 \mathrm{~nm})$, while the fluorescence yield (FY) mode signal is mainly from the bulk. Therefore, sXAS is considered a powerful tool to study surface/interface related issues such as a solid electrolyte interphase (SEI) or a cathode-electrolyte interface (CEI). Combining hXAS and sXAS at multiple probing depths will provide valuable information to understand complicated and inhomogeneous reactions during LIBs operation. The poor penetration power of soft X-rays requires UHV conditions, causing difficulty in performing in situ sXAS experiments, especially for liquid electrolyte cells.

It is quite interesting to note that significant progress has been made recently on designing and applying in situ/ operando cells for sXAS experiments under UHV conditions. For example, Liu et al. ${ }^{13}$ developed an in situ sXAS cell with a modified current collector and polymer electrolyte, as shown Fig. 2e. Interestingly, the strong relaxation effect and a state-of-charge (SOC) gradient were observed in the $\mathrm{LiFePO}_{4}$ electrode, shown in Fig. 8. In contrast, the sXAS results of the $\mathrm{LiCo}_{1 / 3} \mathrm{Ni}_{1 / 3} \mathrm{Mn}_{1 / 3} \mathrm{O}_{2}$ electrodes show uniform SOC across whole electrodes. The cooperation of the electronic and ionic conductivity, the phase transformation mechanism and the mesoscale morphology lead to an observed difference in the charge dynamics of NMC and $\mathrm{LiFePO}_{4}$. It is revealed that electron transportation is the determining factor for $\mathrm{LiFePO}_{4}$ during electrochemical cycling, while the open-circuit 
relaxation time is governed by ionic conductivity. This comparative study demonstrates the power of in situ sXAS for studying charge dynamics in battery electrodes.

As discussed before in the XRD section, the investigation of the depth profile is also considered with XAS. Considering the working principle of XAS, the depth of beam penetration in XAS measurements is highly dependent on the energy level of the incident beam. Unfortunately, the K-edge energy of most of elements used in LIBs (i.e., $3 d$-TM) is below $10 \mathrm{keV}$, which requires a relatively thin sample thickness ( few hundred $\mu \mathrm{m})$. Furthermore, other cell components in the beam pathway, such as window materials or electrolyte, will absorb the beam, and therefore, the cell configuration and materials greatly restricted compared to those in XRD. A RATIX cell might be applicable in some energy ranges, but the thickness of a tubular cell needs to be very thin in a lower energy range. The micro-size beam with high flux is also required. Regardless of such technical difficulties, some efforts have been made to obtain a depth profile for ex situ studies. Orikasa et al. ${ }^{39}$ demonstrated cross-sectional 2D mapping of the absorption energy at the Fe K-edge in $\mathrm{LiFePO}_{4}$ composite electrodes (Fig. 9). Fe K-edge XAS spectra were collected in transmission mode using a CMOS detector with spatial resolution of $10 \mu \mathrm{m}$. 2D Fe Kedge XAS mapping reveals that more significant inhomogeneity of the electrochemical reaction occurred in lower porosity electrodes, which have lower ionic conductivities. This work could further motivate other researchers to develop new cells for in situ depth profile XAS measurements in the future.

\section{In situ X-ray imaging}

Imaging methods provide opportunities for the direct visualization of battery materials and components, and therefore offer straightforward information about the microstructure, morphology, and chemical composition. $\mathrm{X}$-ray microscopy allows for the direct visualization of battery materials and components in a non-invasive manner under in operando conditions. In general, X-ray imaging techniques can be classified into real-space imaging methods and reciprocal space imaging methods depending on the setup of the imaging systems. The reciprocal image methods can achieve a relatively higher spatial resolution because no X-ray optics are needed between the sample and the recorder, removing the optical limits of the spatial resolution of the imaging systems ${ }^{40}$. Based on the data acquisition method, X-ray imaging techniques can also be categorized as full-field $\mathrm{X}$ ray imaging methods and scanning $\mathrm{X}$-ray imaging methods.

Real-space full-field imaging methods are usually superior in terms of the data acquisition speed. The

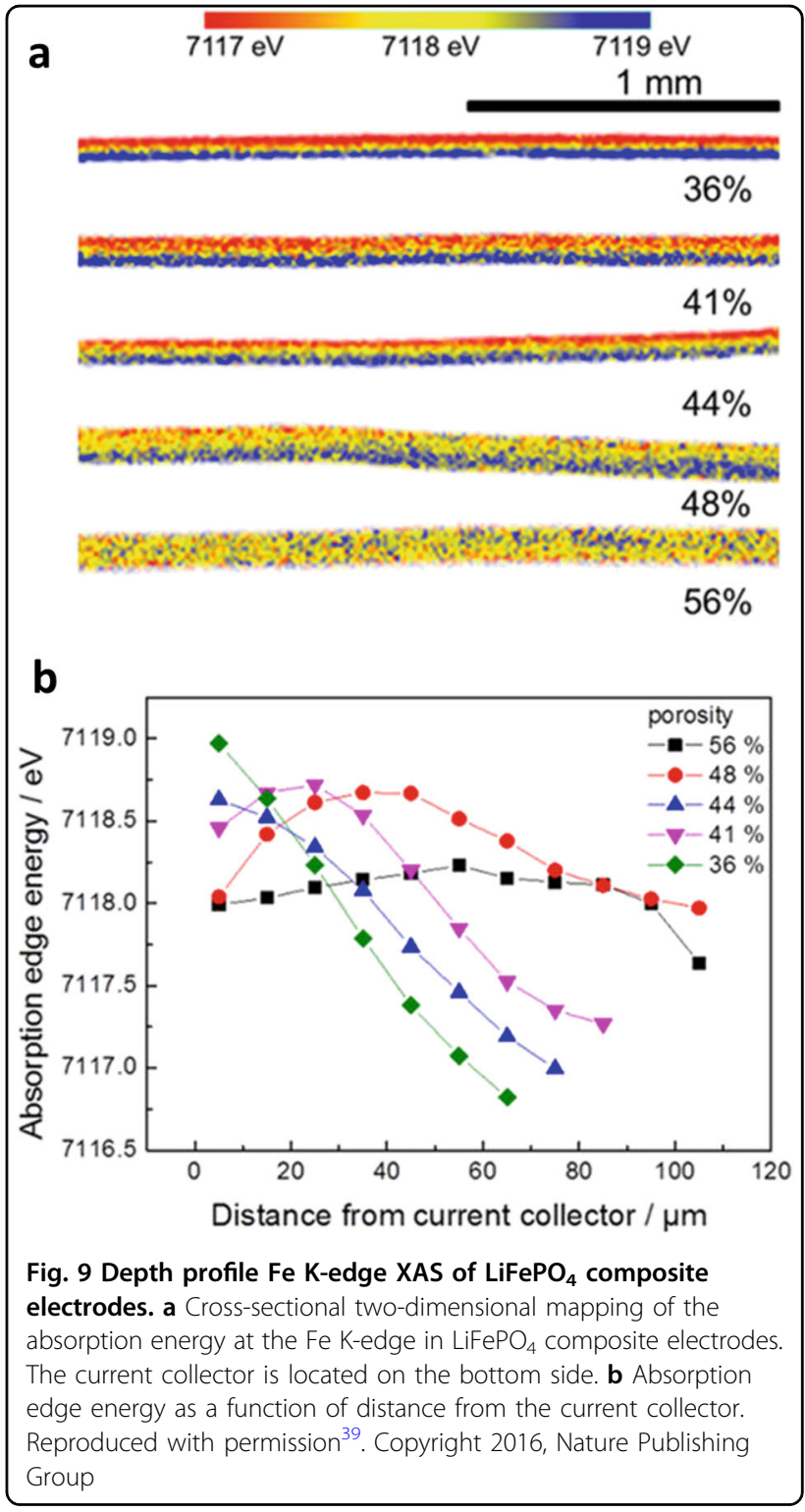

typical exposure time of a single image can be completed in a time frame as short as a few tens of milliseconds at synchrotron light sources, which favors in situ/operando and time-resolved studies. Absorption contrast and phase contrast are the most commonly used mechanisms for full-field X-ray imaging methods. The power of real-space full-field imaging methods to visualize battery materials in real-time operation has been demonstrated by various recent works ${ }^{20,41-49}$. Combined with spectroscopic measurements, full-field X-ray imaging becomes even more powerful for obtaining chemical information in addition to the morphological information. For example, Yang et al. ${ }^{50}$ reported the chemical phase, oxidation state and morphology changes of $\mathrm{Li}_{1.2} \mathrm{Mn}_{0.525} \mathrm{Ni}_{0.175} \mathrm{Co}_{0.1} \mathrm{O}_{2}$ after multiple cycles; Lin et al. ${ }^{51}$ revealed transition metal 


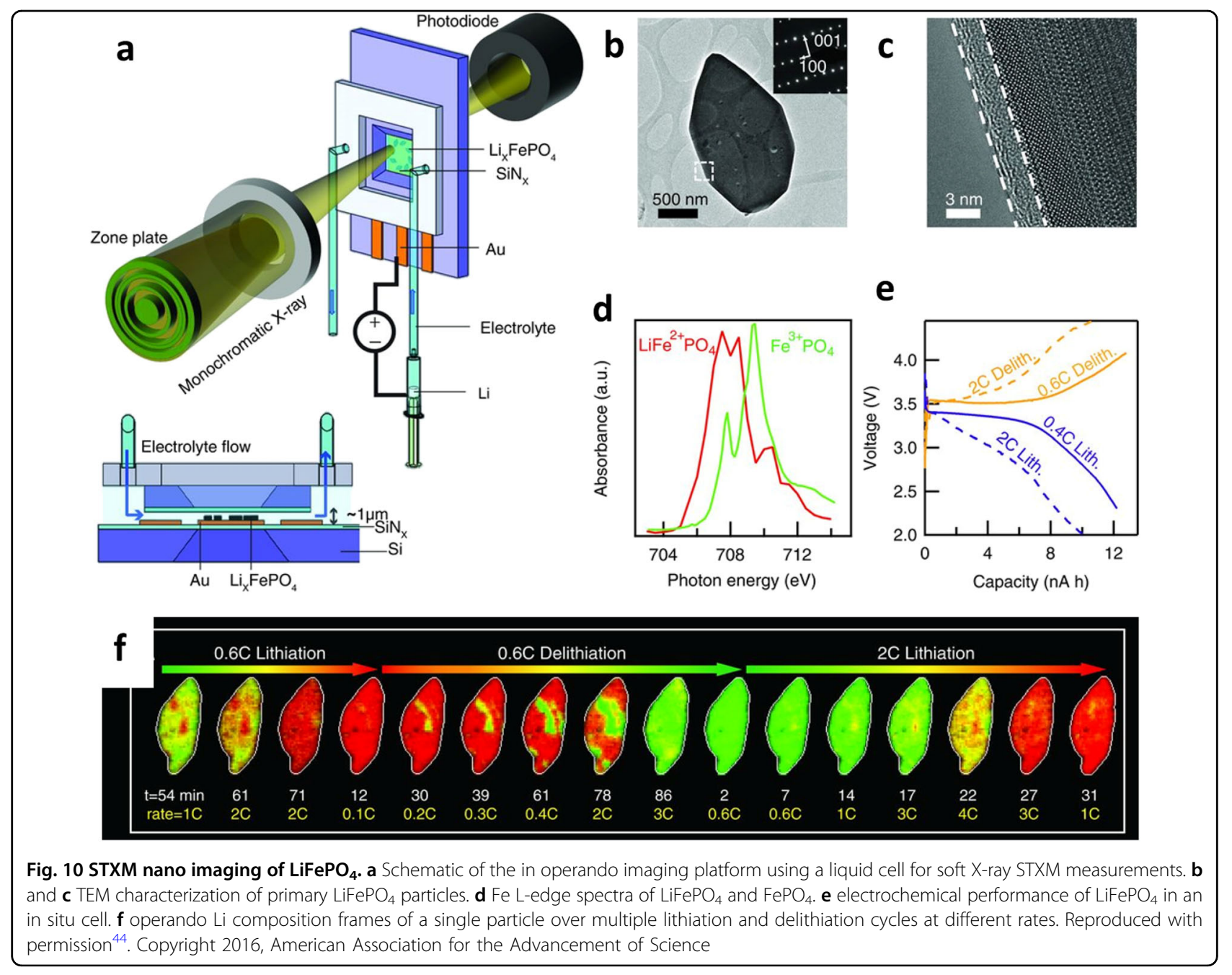

segregation at the subparticle level in $\mathrm{LiNi}_{0.4} \mathrm{Mn}_{0.4} \mathrm{Co}_{0.2} \mathrm{O}_{2}$ cathode material; $\mathrm{Xu}$ et al. ${ }^{52}$ investigated the evolution of chemical and morphological changes of Li-rich $\mathrm{Li}_{2} \mathrm{Ru}_{0.5} \mathrm{Mn}_{0.5} \mathrm{O}_{3}$ cathode materials at the nanoscale upon electrochemical cycling.

Unlike the full-field imaging method, scanning X-ray imaging experiments are performed by using well-focused $\mathrm{X}$-rays to illuminate the sample, which is raster-scanned in the focal plane. Different types of signals (e.g., transmission, fluorescence, diffraction, etc.) can be recorded at each data collecting position to construct the full X-ray images. The major drawback of the scanning method is the relative slower data acquisition speed, which has been greatly improved with the development of more brilliant synchrotron light sources. Scanning imaging techniques for LIB studies have been widely employed in recent years $^{44,53-56}$. One example is the investigation of the spatiotemporal evolution of the $\mathrm{Li}$ composition and insertion rates in $\mathrm{LiFePO}_{4}$ in primary particles by using soft X-ray scanning transmission X-ray microscopy
(STXM) (Fig. 10). Soft X-ray transition metal (TM) Ledge spectra are very sensitive to the valence of $3 d$ TM ions ${ }^{57}$. Lim et al. ${ }^{44}$ designed a soft X-ray in situ liquid cell (with a $\mathrm{Si}_{3} \mathrm{~N}_{4}$ thin film as a window), which can be used in a vacuum chamber during measurement. The dynamics of the nanoscale spatial variation of the Fe valence associated with the lithium composition $\left(\mathrm{Li}_{x} \mathrm{FePO}_{4}\right)$ at different lithiation/delithiation rates was mapped. The results indicate that the nanoscale intraparticle electrochemical reaction inhomogeneity in the phase and composition is rate dependent, and the lithiation and delithiation behavior is remarkably different in $\mathrm{LiFePO}_{4}$. The reaction heterogeneities and compositional non-uniformity at the particle or subparticle scales can be probed and well visualized by in situ/operando X-ray imaging.

Most of the in situ/operando X-ray imaging experiments have been focused on the first or first several charge-discharge cycles only. However, studies of the degradation mechanisms of battery materials inevitably require tracking the electrochemical reaction of the 
batteries for long-term cycling. Since synchrotron beamtime not often granted for prolonged experiments, continuous monitoring of the electrochemical reaction during long-term cycling at synchrotron facilities is not economically feasible. Recently, $\mathrm{Xu}$ and co-workers ${ }^{41}$ developed an in situ setup that is capable of tracking the same particle of a sample after dismounting and remounting it over multiple charge-discharge cycles by intermittent sampling. As seen in Fig. 11, the in situ apparatus is made of a pouch cell attached to a cell holder. The cell holder is mounted on the sample stage with recordable absolute coordinates. As long as the sample is mounted with the same absolute position on the sample stage as the previous measurement, the same sample particle can be relocated. This kind of setup also has the advantage of reducing radiation damage caused by long-term X-ray beam illumination during cycling. Taking advantages of this kind of setup, electrochemical cycling can be done offline (out of the X-ray beam) until the specific cycling state of interest is reached. Under this arrangement, data can be collected as a function of the SOC and/or the number of cycles. As an example, the reaction heterogeneity of $\mathrm{LiCoO}_{2}$ on the sub-micro-scale for a particle at different charge-discharge states and cycle numbers is clearly observed. Inactive domains were observed and redistributed within the particle upon long-term cycling and is directly linked to the degradation behavior of $\mathrm{LiCoO}_{2}$ material. This study also demonstrates that X-ray imaging is capable of investigating the degradation mechanism of battery materials over prolonged cycles.

When the X-ray tomography technique is used, a series of X-ray images at different angles are recorded (the sample is rotated along an axis that is perpendicular to the incident X-rays) and then reconstructed by computer to produce the 3D images ${ }^{58}$. This X-ray tomography technique using synchrotron $\mathrm{X}$-ray sources has been applied to the in situ/operando studies on dynamic 3D morphological changes during electrochemical reactions. Electrode materials that store lithium through conversion or alloying reactions face the major problem of volume changes during lithium insertion and extraction ${ }^{42,59,60}$. The in operando study of dynamic changes in the sample morphology in 3D provides valuable information for the fundamental understanding of the lithium (or other alkali ions) storage mechanisms of these materials, as well as insightful information for material design. Several good examples of using in situ/operando X-ray tomography for battery material studies are shown in the work of Villevieille et al. $^{49}$ regarding the influence of particle morphology on the alloying phase transformation process of an $\mathrm{Sb} / \mathrm{TiSb}_{2}$ electrode, and in the work of Wang et al. ${ }^{14}$ regarding three-dimensional structural/chemical evolution of a tin anode electrode material for sodium-ion batteries. A more detailed study using a tomographic

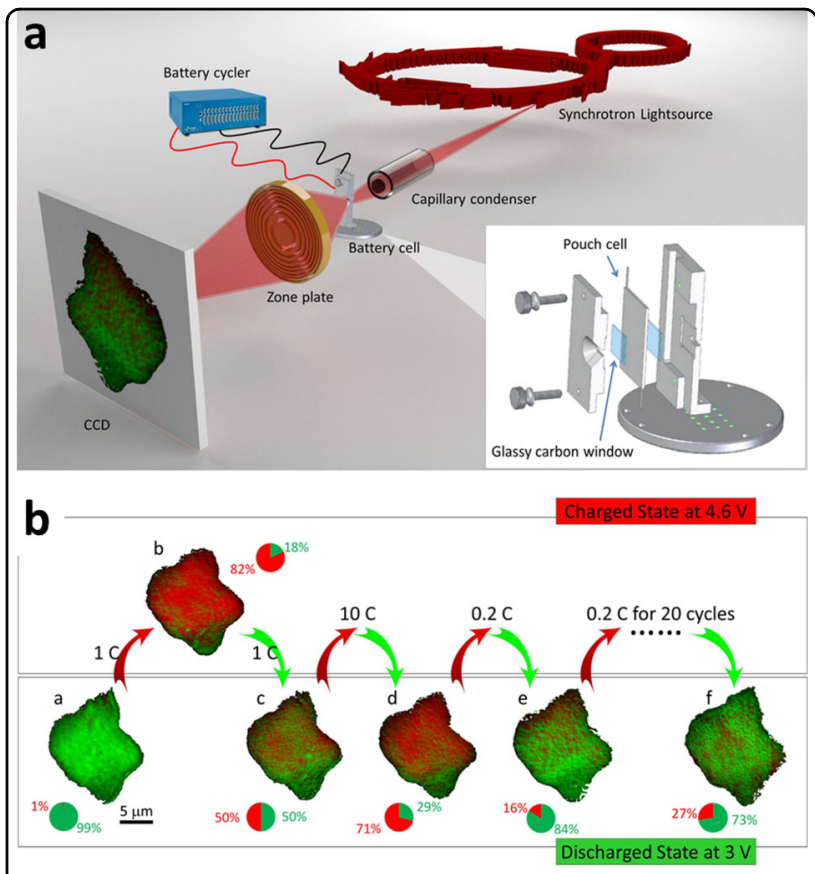

Fig. $11 \mathrm{X}$-ray imaging to investigate the degradation mechanism of $\mathrm{LiCoO}_{2}$ over prolonged cycles. a Schematic illustration of the setup used for the in situ microspectroscopy study of LIB. The lower right inset shows a detailed view of the developed pouch cell system that allowed the in situ study of the long-term cycling behavior of battery materials. $\mathbf{b}$ In situ monitoring of the chemical heterogeneity in a single particle of $\mathrm{LiCoO}_{2}$ during battery cycling. Reproduced with permission ${ }^{41}$. Copyright 2017, American Chemical Society

technique for an in operando experiment was reported by Ebner et al. ${ }^{42}$ as shown in (Fig. 12). They used X-ray tomography to visualize the morphological changes in a conversion/alloying-type $\mathrm{SnO}$ anode during battery operation, and discussed the origins of electrochemical and mechanical degradation associated with such volume changes. The chemical reaction of $\mathrm{SnO}$ during lithium insertion (discharge) and extraction (charge) can be tracked by monitoring the attenuation coefficient of the electrode. The tomographic analysis provided 3D chemical and morphological information within a sample particle. The phase transformation, crack creation and growth, and electrode deformation can be clearly observed, offering direct visualization associated with the degradation of battery performance. The application of Xray tomography can be extended to lithium metal anode research; although, it is well known that lithium is difficult to characterize by using $\mathrm{X}$-ray-based techniques due to the weak interaction between lithium and the X-ray photons. A recent publication by Guillaume et al. shows promising results proving that $\mathrm{X}$-ray tomography is useful for investigating the morphology and structural changes of lithium metal anodes ${ }^{61}$. Overall, X-ray tomography enables the investigation of dynamic changes in a sample 


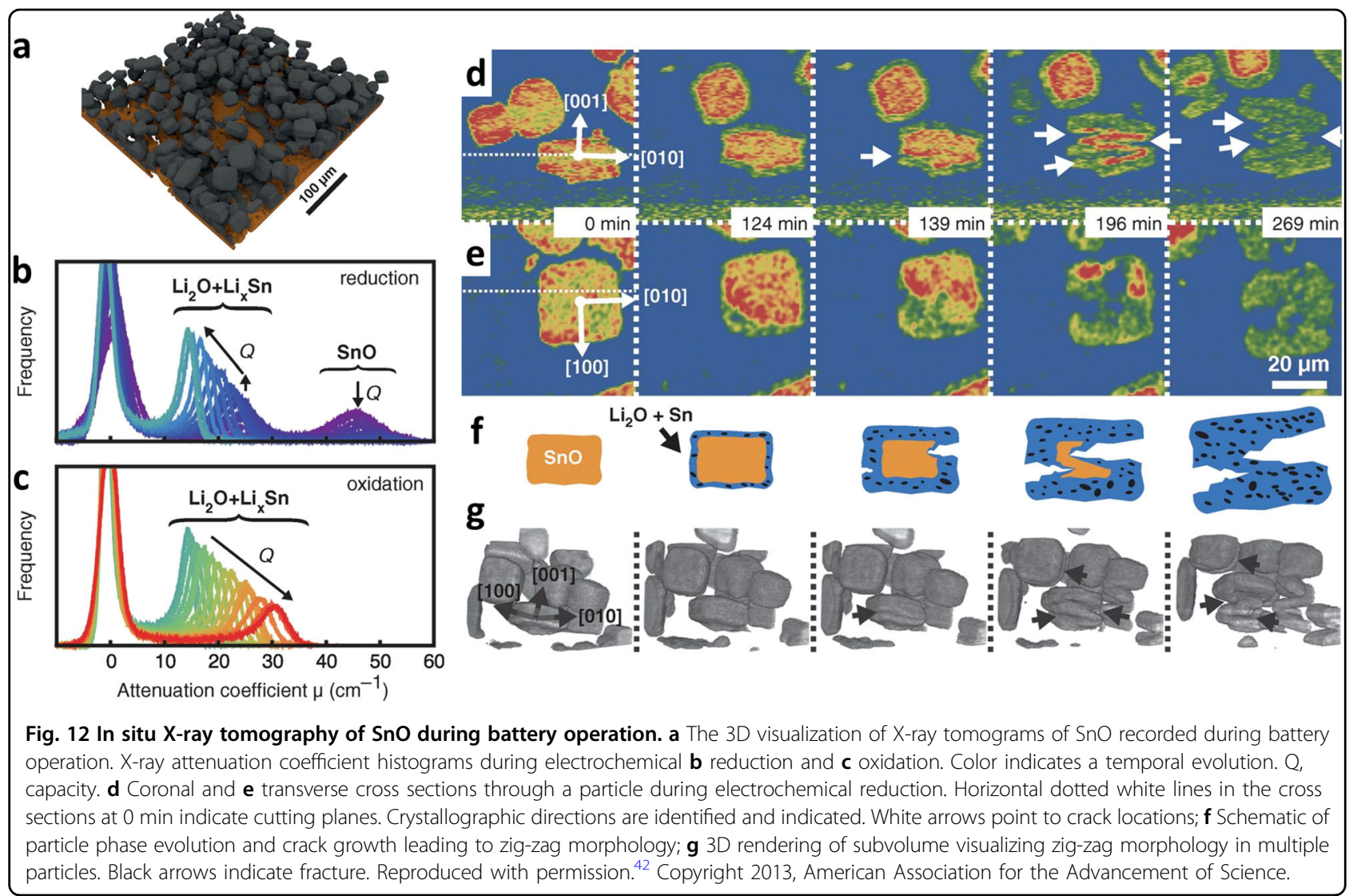

in real batteries and, therefore, opens a new avenue for the rational design of battery materials.

\section{Perspectives}

Significant progress toward developing in situ/operando techniques using an advanced synchrotron X-ray source has been made, and various in situ cell designs for LIB studies have been reported recently. The studies of in situ/operando XRD, PDF, XAS, and imaging techniques highlighted in this review can be used as good references to guide future development of in situ/ operando experiments, and suitable in situ electrochemical cells for such experiments as well as the selection of appropriate X-ray techniques for certain research purposes.

The combination of different characterization techniques is important to obtain a more complete picture and more comprehensive information about the structural changes in LIB materials. For example, although synchrotron-based X-ray imaging techniques can provide morphological and chemical information at wide length scales, from tens of $\mathrm{nm}$ to the $\mathrm{mm}$ scale, imaging at the atomic-length scale (sub or few $\mathrm{nm}$ ) still requires transmission electron microscopy (TEM) techniques. Nuclear magnetic resonance (NMR) and mass spectrometry (MS) can also provide important complementary information to the X-ray techniques. For further development of in situ/operando X-ray techniques, high-speed data collection and the automated analysis of large amount of data (data mining) are quite important, and we hope this review is helpful for these aspects.

\section{Acknowledgements}

The work at Brookhaven National Lab was supported by the U.S. Department of Energy, the Assistant Secretary for Energy Efficiency and Renewable Energy, Vehicle Technologies Office (BMR project including the VTO Battery 500) under Contract Number DE-SC0012704. The work at the Institute of Physics, Chinese Academy of Sciences was supported by funding from the National Key R\&D Program of China (Grants No. 2017YFB0102004) and "Thousand Talents Program for Young Scientists".

\section{Author details}

${ }^{1}$ Chemistry Division, Brookhaven National Laboratory, Upton NY11973, United States. ${ }^{2}$ Beijing National Laboratory for Condensed Matter Physics, Institute of Physics, Chinese Academic of Science, Beijing 100190, P.R. China

\section{Conflict of interest}

The authors declare that they have no conflict of interest.

\section{Publisher's note}

Springer Nature remains neutral with regard to jurisdictional claims in published maps and institutional affiliations.

Received: 20 December 2017 Revised: 25 April 2018 Accepted: 15 May 2018.

Published online: 3 July 2018 


\section{References}

1. Armand, M. \& Tarascon, J. M. Building better batteries. Nature 451, 652-657 (2008).

2. Lin, F. et al. Synchrotron X-ray analytical techniques for studying materials electrochemistry in rechargeable batteries. Chem. Rev. 117, 13123-13186 (2017).

3. Harks, P. P. R. M. L., Mulder, F. M. \& Notten, P. H. L. In situ methods for Li-ion battery research: a review of recent developments. J. Power Sources $\mathbf{2 8 8}$ 92-105 (2015).

4. Yang, J. et al. In situ analyses for ion storage materials. Chem. Soc. Rev. 45 5717-5770 (2016)

5. Grey, C. P. \& Tarascon, J. M. Sustainability and in situ monitoring in battery development. Nat. Mater. 16, 45-56 (2017).

6. Hu, Y. Y. et al. Origin of additional capacities in metal oxide lithium-ion battery electrodes. Nat. Mater. 12, 1130-1136 (2013).

7. Yoon, W. S. et al. Investigation of the charge compensation mechanism on the electrochemically Li-ion deintercalated $\mathrm{Li}_{1-x} \mathrm{CO}_{1 / 3} \mathrm{Ni}_{1 / 3} \mathrm{Mn}_{1 / 3} \mathrm{O}_{2}$ electrode system by combination of soft and hard X-ray absorption spectroscopy. J. Am. Chem. Soc. 127, 17479-17487 (2005).

8. Yoon, W. S., Grey, C. P., Balasubramanian, M., Yang, X. Q. \& McBreen, J. In situ Xray absorption spectroscopic study on $\mathrm{LiNi}_{0.5} \mathrm{Mn}_{0.5} \mathrm{O}_{2}$ cathode material during electrochemical cycling. Chem. Mater. 15, 3161-3169 (2003).

9. Kim, $\mathrm{H}$. et al. New insight into the reaction mechanism for exceptional capacity of ordered mesoporous $\mathrm{SnO}_{2}$ electrodes via synchrotron-based X-ray analysis. Chem. Mater. 26, 6361-6370 (2014).

10. Gauthier, M. et al. Electrode-electrolyte interface in Li-ion batteries: current understanding and new insights. J. Phys. Chem. Lett. 6, 4653-4672 (2015).

11. Borkiewicz, O. J. et al. The AMPIX electrochemical cell: a versatile apparatus for in situ X-ray scattering and spectroscopic measurements. J. Appl. Crystallogr. 45, 1261-1269 (2012).

12. Liu, H. et al. A radially accessible tubular in situ X-ray cell for spatially resolved operando scattering and spectroscopic studies of electrochemical energy storage devices. J. Appl. Crystallogr. 49, 1665-1673 (2016).

13. Liu, X. S. et al. Distinct charge dynamics in battery electrodes revealed by in situ and operando soft X-ray spectroscopy. Nat. Commun. 4, 2568 (2013).

14. Wang, J., Eng, C., Chen-Wiegart, Y. C. \& Wang, J. Probing three-dimensional sodiation-desodiation equilibrium in sodium-ion batteries by in situ hard $\mathrm{X}$-ray nanotomography. Nat. Commun. 6, 7496 (2015).

15. Zhou, Y. N. et al. High-rate charging induced intermediate phases and structural changes of layer-structured cathode for lithium-ion batteries. Adv. Energy Mater. 6, 1600597 (2016).

16. Liu, $\mathrm{H}$. et al. Capturing metastable structures during high-rate cycling of $\mathrm{LiFePO}_{4}$ nanoparticle electrodes. Science 344, 1252817 (2014).

17. Muhammad, S. et al. Evidence of reversible oxygen participation in anomalously high capacity Li- and Mn-rich cathodes for Li-ion batteries. Nano Energy 21, 172-184 (2016).

18. Yang, Z. Z. et al. In situ high-energy synchrotron $X$-ray diffraction studies and first principles modeling of alpha- $\mathrm{MnO}_{2}$ electrodes in $\mathrm{Li}_{-} \mathrm{O}_{2}$ and $\mathrm{Li}$-ion coin cells. J. Mater. Chem. A 3, 7389-7398 (2015).

19. Storm, M. M., Johnsen, R. E., Younesi, R. \& Norby, P. Capillary based Li-air batteries for in situ synchrotron X-ray powder diffraction studies. J. Mater. Chem. A 3, 3113-3119 (2015).

20. Wang, J. J., Chen-Wiegart, Y. C. K. \& Wang, J. In operando tracking phase transformation evolution of lithium iron phosphate with hard $\mathrm{X}$-ray microscopy. Nat. Commun. 5, 4570 (2014).

21. Liu, Q. et al. Rate-dependent, Li-ion insertion/deinsertion behavior of LiFePo4 cathodes in commercial 18650 LiFePo4cells. Acs Appl. Mater. Inter. 6 3282-3289 (2014)

22. $\mathrm{Yu}, \mathrm{X}$. Q. et al. Direct observation of the redistribution of sulfur and polysufides in Li-S batteries during the first cycle by in situ X-ray fluorescence microscopy. Adv. Energy Mater. 5, 1500072 (2015).

23. Seeck, O. H., \& Murphy, B . X-Ray Diffraction: Modern Experimental Techniques. (CRC Press: New York, 2015).

24. Toby, B. H. \& Dreele, R. B. V. GSAS-II: the genesis of a modern open-source all purpose crystallography software package. J. Appl. Crystallogr. 46, 544-549 (2013).

25. Qiu, X., Thompson, J. W. \& Billinge, S. J. L. PDFgetX2: a GUI-driven program to obtain the pair distribution function from X-ray powder diffraction data. J. Appl. Crystallogr. 37, 678 (2004)

26. Calvin, S. XAFS for Everyone. (CRC Press, New York, 2013).
27. Ravel, B. \& Newville, M. ATHENA, ARTEMIS, HEPHAESTUS: data analysis for X-ray absorption spectroscopy using IFEFFIT. J. Synchrotron Radiat. 12, 537-541 (2005).

28. Liu, Y. et al. TXM-Wizard: a program for advanced data collection and evaluation in full-field transmission X-ray microscopy. J. Synchrotron Radiat. 19, 281-287 (2012)

29. Zhou, Y. N. et al. Tuning charge-discharge induced unit cell breathing in layerstructured cathode materials for lithium-ion batteries. Nat. Commun. 5, 5381 (2014).

30. Wiaderek, K. M. et al. Comprehensive insights into the structural and chemical changes in mixed-anion FeOF electrodes by using operando PDF and NMR spectroscopy. J. Am. Chem. Soc. 135, 4070-4078 (2013).

31. Hua, $\mathrm{X}$. et al. Comprehensive study of the $\mathrm{CuF}_{2}$ conversion reaction mechanism in a lithium ion battery. J. Phys. Chem. C. 118, 15169-15184 (2014).

32. Borkiewicz, O. J., Chapman, K. W. \& Chupas, P. J. Mapping spatially inhomogeneous electrochemical reactions in battery electrodes using high energy $\mathrm{X}$ rays. Phys. Chem. Chem. Phys. 15, 8466-8469 (2013).

33. Northrup, P., Leri, A. \& Tappero, R. Applications of "tender" energy (1-5 kev) Xray absorption spectroscopy in life sciences. Protein Pept. Lett. 23, 300-308 (2016).

34. Iwasawa, Y., Asakura, K. \& Tada, M. XAFS Techniques for Catalyst, Nanomaterials, and Surfaces. (Springer International Publishing, Switzerland, 2017).

35. Stohr, J. NEXAFS Spectroscopy. (Springer: New York, 1992.

36. $\mathrm{Yu}, \mathrm{X}$. Q. et al. Understanding the rate capability of high-energy-density Li-rich layered $\mathrm{Li}_{1.2} \mathrm{Ni}_{0.15} \mathrm{CO}_{0.1} \mathrm{Mn}_{0.55} \mathrm{O}_{2}$ cathode materials. Adv. Energy Mater. 4 1300950 (2014).

37. Muller, O., Nachtegaal, M., Just, J., Lutzenkirchen-Hecht, D. \& Frahm, R. Quickexafs setup at the superxas beamline for in situ X-ray absorption spectroscopy with 10 ms time resolution. J. Synchrotron Radiat. 23, 260-266 (2016).

38. Prestipino, C., Mathon, O., Hino, R., Beteva, A. \& Pascarelli, S. Quick-EXAFS implementation on the general purpose exafs beamline at esif. J. Synchrotron Radiat. 18, 176-182 (2011)

39. Orikasa, $Y$. et al. Ionic conduction in lithium ion battery composite electrode governs cross-sectional reaction distribution. Sci. Rep. 6, 26382 (2016).

40. Miao, J. W., Ishikawa, T., Robinson, I. K. \& Murnane, M. M. Beyond crystallography: diffractive imaging using coherent $X$-ray light sources. Science $\mathbf{3 4 8}$, 530-535 (2015).

41. $\mathrm{Xu}, \mathrm{Y} . \mathrm{H}$. et al. In situ visualization of state-of-charge heterogeneity within a $\mathrm{LiCOO}_{2}$ particle that evolves upon cycling at different rates. Acs Energy Lett. 2, 1240-1245 (2017).

42. Ebner, M., Marone, F., Stampanoni, M. \& Wood, V. Visualization and quantification of electrochemical and mechanical degradation in $\mathrm{Li}$ ion batteries. Science 342, 716-720 (2013).

43. Weker, J. N. et al. In situ nanotomography and operando transmission X-ray microscopy of micron-sized ge particles. Energy Environ. Sci. 7, 2771-2777 (2014).

44. Lim, J. et al. Origin and hysteresis of lithium compositional spatiodynamics within battery primary particles. Science 353, 566-571 (2016).

45. Wang, J. J., Chen-Wiegart, Y. C. K. \& Wang, J. In situ three-dimensional synchrotron $X$-ray nanotomography of the (de) lithiation processes in tin anodes. Angew. Chem. Int. Ed. 53, 4460-4464 (2014).

46. Chao, S. C. et al. In situ transmission X-ray microscopy study on working SnO anode particle of Li-ion batteries. J. Electrochem. Soc. 158, A1335-A1339 (2011).

47. Chao, S. C. et al. Study on microstructural deformation of working sn and SnSb anode particles for Li-ion batteries by in situ transmission X-ray microscopy. J. Phys. Chem. C. 115, 22040-22047 (2011).

48. Brushett, F. R., Trahey, L., Xiao, X. H. \& Vaughey, J. T. Full-field synchrotron tomography of nongraphitic foam and laminate anodes for lithium-ion batteries. Acs Appl. Mater. Inter. 6, 4524-4534 (2014).

49. Villevieille, C., Sasaki, T. \& Novak, P. Novel electrochemical cell designed for operando techniques an dimpedance sudies. RSC Adv. Influence of conversion material morphology on electrochemistry studied with operando X-ray tomography and diffraction. Adv. Mater. 4, 6782-6789 (2014).

50. Yang, F. F. et al. Nanoscale morphological and chemical changes of high voltage lithium manganese rich nmc composite cathodes with cycling. Nano Lett. 14, 4334-4341 (2014).

51. Lin, F. et al. Metal segregation in hierarchically structured cathode materials for high-energy lithium batteries. Nat. Energy 1, 15004 (2016).

52. $\mathrm{Xu}, \mathrm{Y}$. H. et al. Structural integrity-searching the key factor to suppress the voltage fade of Li-rich layered cathode materials through $3 \mathrm{~d}$ X-ray imaging and spectroscopy techniques. Nano Energy 28, 164-171 (2016). 
53. Li, Y. Y. et al. Dichotomy in the lithiation pathway of ellipsoidal and platelet $\mathrm{LiFePO}_{4}$ particles revealed through nanoscale operando state-of-charge imaging. Adv. Funct. Mater. 25, 3677-3687 (2015).

54. Li, Y. Y. et al. Current-induced transition from particle-by-particle to concurrent intercalation in phase-separating battery electrodes. Nat. Mater. 13, 1149-1156 (2014).

55. Ohmer, N. et al. Phase evolution in single-crystalline $\mathrm{LiFePO}_{4}$ followed by in situ scanning $\mathrm{X}$-ray microscopy of a micrometre-sized battery. Nat. Commun. 6, 6045 (2015).

56. Yu, Y. S. et al. Dependence on crystal size of the nanoscale chemical phase distribution and fracture in $\mathrm{Li}_{x} \mathrm{FePO}_{4}$. Nano Lett. 15, 4282-4288 (2015).

57. Asakura, D. et al. Material/element-dependent fluorescence-yield modes on soft X-ray absorption spectroscopy of cathode materials for Li-ion batteries. AIP Adv. 6, 035105 (2016).
58. Liu, Y. J., Kiss, A. M., Larsson, D. H., Yang, F. F. \& Pianetta, P. To get the most out of high resolution $X$-ray tomography: a review of the post-reconstruction analysis. Spectrochim. Acta B 117, 29-41 (2016).

59. Poizot, P., Laruelle, S., Grugeon, S., Dupont, L. \& Tarascon, J. M. Nano-sized transition-metaloxides as negative-electrode materials for lithium-ion batteries. Nature 407, 496-499 (2000).

60. He, Y. et al. Shape evolution of patterned amorphous and polycrystalline silicon microarray thin film electrodes caused by lithium insertion and extraction. J. Power Sources 216, 131-138 (2012).

61. Tonin, G. et al. Multiscale characterization of a lithium/sulfur battery by coupling operando X-ray tomography and spatially-resolved diffraction. Sci. Rep. 7 , 2755 (2017). 